\title{
Classes of Admissible Exchange- Correlation Density Functionals for Pure Spin and Angular Momentum States
}

\author{
A. L. TCHOUGRÉEFF, ${ }^{1,2,3}$ J. G. ÁNGYÁN ${ }^{4}$ \\ ${ }^{1} J A R A$, Institute of Inorganic Chemistry, RWTH Aachen, Germany \\ ${ }^{2}$ Poncelet Laboratory, Independent University of Moscow, Moscow Center for Contunous \\ Mathematical Education, 119991 Moscow, Russia \\ ${ }^{3}$ Division of Electrochemistry, Department of Chemistry, Moscow State University, \\ 119991 Moscow, Russia \\ ${ }^{4}$ CRM2, Nancy-University, CNRS, B.P. 239, Vandoeuvre-lès-Nancy, France
}

Received 18 January 2009; accepted 17 February 2009

Published online 3 June 2009 in Wiley InterScience (www.interscience.wiley.com).

DOI 10.1002/qua.22201

\begin{abstract}
We analyze various approaches to construct exchange-correlation functionals which are able to describe states of definite spin multiplicity in the density functional theory (DFT) realm and outline the characteristics of possible functionals consistent with the Kohn-Sham theory. To achieve this goal the unitary group technique is applied to label many-electron states of definite total spin and to calculate the corresponding analogs of the Roothaan coupling coefficients. The possibility of using range separated Coulomb potential of electron-electron interaction for constructing functionals discriminating multiplet states in the $d$-shells is explored and a tentative system of state-specific functionals, covering nontrivial correlations in $d$-shells of transition metal ions, is proposed for the $\mathrm{Fe}^{2+}$ ions. () 2009 Wiley Periodicals, Inc. Int J Quantum Chem 110: 454-475, 2010
\end{abstract}

Key words: DFT; multiplet states; correlation functional; exchange functional; Young tableaux

\section{Introduction}

A lthough the density functional theory (DFT) based methods of modeling electronic structure of molecules and solids widely proliferate dur-

Correspondence to: A. Tchougreeff; e-mail: tch@elch.chem.msu.ru Contract grant sponsor: RFBR.

Contract grants numbers: 07-03-01128, 08-03-00414. ing last decades [1-3], the problem of consistent description of transition metal and rare earth compounds with open $d$ - and $f$-shells, respectively, remains a still unresolved, challenging problem in this framework [4]. One of the main reasons for this failure of the DFT is that the multiplet spin/orbital momentum states are generally not easily described within the DFT paradigm. The source of that intimate "unfriendliness" of the DFT to the multiplet states lays in the "oversymmetry" of the fundamen- 
tal quantity pertaining to the realm of DFT: the one-electron density. As it has been demonstrated many times, states of different total spin and/or spatial symmetry may produce equal one-electron densities. The complication arising from this is the impossibility to distinguish the nature of the ground state on the basis of the total density only: although only one of say two functions represents the ground state, that is, the exact energies of the involved states may be different, they turn out to be degenerate in the DFT context. In other terms, if the same densities are fed to the "universal" density functional implied by the DFT, it is going to produce the same value of the electronic energy for states whose exact energies are different. Of course the latter remark may be opposed by noting that the "universal" functional is going to output the ground state energy only, but in this case it is not clear how other important pieces of information concerning the nature of this ground state (e.g., its spin multiplicity) can be extracted from such an answer.

This situation certainly requires some clarification which is addressed in this article. To do so, we give below a brief description of relevant elements of the electronic structure theory (Section 2). Then we consider an archetypical example of the problems encountered by the DFT while trying to reproduce correct spin properties of many-electron systems (Section 3). Then we propose a general scheme allowing to include states of definite spin in the DFT theory (Section 4). This, however, does not solve the problem of the multiple states in the open $d$ - and $f$-shells of the transition and rare earth ions. For this end, we explore in Section 6 the possibility to circumvent these problems with use of the short/ long range separation of Coulomb interaction between electrons and propose in Section 5 some conceivable state-dependent definition of exchange-correlation functionals capable to reproduce the energies of nontrivially correlated manyelectronic states in the $d$-shell of the $\mathrm{Fe}^{2+}$ ions.

\section{Theoretical Background}

\subsection{ELECTRONIC DISTRIBUTION}

The main idea of the DFT is to reduce the description of entire electronic structure to a single quantity: the one-electron density, the diagonal part of the one-electron density matrix. The possibility of such a reduction is proven by the Hohenberg-
Kohn theorems [5] which state an existence of a universal one-to-one correspondence between oneelectron external potential and the one-electron density in that sense that not only the one-electron potential acting upon a given number of electrons uniquely defines the ground state of such a system, that is, its wave function and thus the one-electron density, but also that for each given density integrating to a given number of electrons $\mathrm{N}$ a oneelectron potential yielding that given density can be uniquely defined from the density. The "density only" formulation of the electronic structure problem, even if it is practically achieved, leaves unanswered an important question of the nature of the ground state thus obtained, for example, about its total spin (or other symmetry features).

Incidentally, the symmetry properties of quantum states, like total spin are easier expressed in terms of wave functions (see Ref. [6].) so it would be practical to consider tentative relation between the wave function and density only pictures of the electronic structure. The required relation can be established with use of the reduced one- and twoelectron density matrices as much simpler objects than the wave functions, providing equivalent description of electronic structure. The reduced density matrices respectively depend on two $\left(x, x^{\prime}\right)$ and four $\left(x_{1} x_{2}, x_{1}{ }^{\prime} x_{2}{ }^{\prime}\right)$ coordinates:

$$
\begin{aligned}
\rho^{(1)}\left(x ; x^{\prime}\right)= & N \int \Psi^{*}\left(x, x_{2}, \ldots x_{N}\right) \\
& \left.\times \Psi x^{\prime}, x_{2}, \ldots, x_{N}\right) d x_{2} \ldots d x_{N} \\
\rho^{(2)}\left(x_{1} x_{2} ; x^{\prime}{ }_{1} x^{\prime}{ }_{2}\right)= & \frac{N(N-1)}{2} \int \Psi^{*}\left(x_{1}, x_{2}, x_{3}, \ldots x_{N}\right) \\
& \times \Psi\left(x^{\prime}{ }_{1}, x^{\prime}{ }_{2}, x_{3}, \ldots, x_{N}\right) d x_{3} \ldots . d x_{N},
\end{aligned}
$$

where the composite electronic coordinate $x$ represents a pair $(\mathbf{r}, s)$ of three dimensional radius vector $r$ of an electron and of the discrete variable $s$ taking either of the two allowed values $\pm \hbar / 2$ of the projection of electronic spin. The transition to the description in terms of reduced density matrices is itself a significant simplification (although being absolutely exact). The one-electron density implied by the DFT theory appears then as a result of further reduction of Eq. (1):

$$
\rho(\mathbf{r})=\sum_{s} \rho^{(1)}(\mathbf{r s} ; \mathbf{r} s)
$$


Thus according to the DFT paradigm the oneelectron density which depends on one spatial radius-vector must be able to serve as an equivalent substitute to the $\mathrm{N}$-electronic wave function dependent on $N$ radius vectors and $N$ more spin projections of all electrons involved. The obvious loss of information which takes place by going from Eq. (1) to Eq. (2) - we remind that going from the wave function $\psi\left(x_{1}, x_{2}, x_{3}, \ldots x_{N}\right)$ to the reduced density matrices by Eq. (1) does not produce any loss of at least important information-must be compensated by the "universal" and "exact" density functional which is in general unknown.

\subsection{ELECTRONIC ENERGY}

Leaving aside the "ideal" DFT using the unknown "universal" and "exact" functional of the density Eq. (2) and turning to pragmatic methods pertaining to the DFT realm needs some approximate expressions for the energy presented as a functional of the density Eq. (2) only. In the wave function and in the equivalent reduced density matrix formulations the energy has the form:

$$
E=\left\langle\hat{T}_{e}\right\rangle+\left\langle\hat{V}_{n e}(\{\mathbf{R}\})\right\rangle+\left\langle\hat{V}_{e e}\right\rangle+V_{n n}(\{R\})
$$

where $\{\mathbf{R}\}$ stands for the set of radius-vectors of all nuclei inducing the electrostatic potential external to the electrons of the system. In the coordinate representation, the above averages acquire familiar appearance:

$$
\begin{gathered}
\left\langle\hat{T}_{e}\right\rangle=-\frac{1}{2} \sum_{s} \int_{\mathbf{r}=\mathbf{r}^{\prime}} \Delta^{\prime} \rho^{(1)}\left(\mathbf{r} s ; \mathbf{r}^{\prime} s\right) d \mathbf{r} \\
\left\langle\hat{V}_{n e}(\{\mathbf{R}\})\right\rangle=\sum_{i} Z_{i} \int \frac{\rho(\mathbf{r}) d \mathbf{r}}{\left|\mathbf{R}_{i}-\mathbf{r}\right|} \\
\left\langle\hat{V}_{e e}\right\rangle=\frac{1}{2} \sum_{s s^{\prime}} \iint \frac{\rho^{(2)}\left(\mathbf{r} s, \mathbf{r}^{\prime} s^{\prime} ; \mathbf{r} s, \mathbf{r}^{\prime} s^{\prime}\right)}{\left|\mathbf{r}-\mathbf{r}^{\prime}\right|} d \mathbf{r} d \mathbf{r}^{\prime} \\
V_{n n}(\{\mathbf{R}\})=\frac{1}{2} \sum_{i \neq j} \frac{Z_{i} Z_{j}}{\left|\mathbf{R}_{i}-\mathbf{R}_{j}\right|} ; \text { where } \\
\Delta^{\prime}=\frac{\partial^{2}}{\partial x^{\prime 2}}+\frac{\partial^{2}}{\partial y^{\prime 2}}+\frac{\partial^{2}}{\partial z^{\prime 2}}
\end{gathered}
$$

where the expressions Eq. (4) are assumed to be specific for a given geometry $\{\mathbf{R}\}$ and for an elec- tronic state described by the $\mathrm{N}$-electronic wave function $\psi=\psi\left(x_{1}, \ldots, x_{N}\right)$ used to define the density matrices Eq. (1). The first row in Eq. (4) is the kinetic energy of electrons, the second row is the energy of Coulomb attraction of electrons to nuclei, the third row is the energy of interelectronic repulsion, and the fourth one is the energy of Coulomb repulsion of the nuclei, which does not depend on the electronic density/wavefunction.

In the above expressions Eqs. (3) and (4), only the average of the nuclear potential $\hat{V}_{n e}$ is exactly a functional of the required form: that of the oneelectron density Eq. (2). All other terms in Eqs. (3) and (4) require further consideration. It applies similarly to the remaining one- and two-electron contributions to the energy. As for the one-electron term, the kinetic energy requires knowledge of the one-electron density matrix Eq. (1) rather than its diagonal part Eq. (2) although effectively in a narrow range of spatial separations $\mathbf{r}-\mathbf{r}^{\prime}$, which must be sufficient to determine the second derivative. The attempts to avoid this bottleneck and to obtain pragmatic DFT methods brought Kohn and Sham [7] to their famous orbital construct which allowed them to express the kinetic energy in terms of some single-determinant wave function yielding by definition the required (exact) one-electron density. Then the kinetic energy is calculated as one of the system of noninteracting electrons described by a single determinant built of KS orbitals.

\subsection{ELECTRONIC DENSITY AND ELECTRONIC ENERGY DECOMPOSITIONS}

Although the Kohn-Sham construct offers an efficient technique to handle the difficult kinetic energy problem and provide a very good first approximation to it, the representation of the electronelectron interaction energy in terms of the oneelectron density (and possibly further parameters derived from the KS determinant) remains the central problem on modern density functional theory. Generally, calculating the Coulomb electron-electron energy (3-rd row of Eq. (4)) requires knowledge of the two-electron density matrix. According to [8] it decomposes:

$$
\begin{array}{r}
\rho^{(2)}\left(x_{1}, x_{2} ; x^{\prime}{ }_{1}, x^{\prime}{ }_{2}\right)=\frac{1}{2}\left|\begin{array}{ll}
\rho^{(1)}\left(x_{1} ; x^{\prime}{ }_{1}\right) & \rho^{(1)}\left(x_{2} ; x^{\prime}{ }_{1}\right) \\
\rho^{(1)}\left(x_{1} ; x^{\prime}{ }_{2}\right) & \rho^{(1)}\left(x_{2} ; x^{\prime}{ }_{2}\right)
\end{array}\right| \\
-\chi\left(x_{1} ; x_{2} ; x_{1}{ }_{1} ; x^{\prime}{ }_{2}\right),
\end{array}
$$

where the first (determinant) term corresponds to the part of the two-particle density matrix which 
can be accounted for even in the independent electrons approximation. The second term in Eq. (5) the cumulant of the two-particle density matrix-is responsible for deviation of electrons' behavior from the independent electron model, that is, for their Coulomb correlations. The Coulomb interaction of electrons Eq. (4) can be decomposed to contributions associated to the terms of the above twoparticle density matrix decomposition Eq. (5):

$$
\begin{gathered}
\left\langle V_{e e}\right\rangle=E_{\mathrm{H}}+\bar{E}_{x \mathrm{c}} ; \\
\bar{E}_{x \mathrm{c}}=\bar{E}_{x}+\bar{E}_{\mathrm{c}}
\end{gathered}
$$

by singling out first the "classical" part of the Coulomb interaction energy, the Hartree energy:

$$
\begin{aligned}
E_{\mathrm{H}}=\frac{1}{2} \sum_{s s^{\prime}} \int \frac{\rho^{(1)}(\mathbf{r} s, \mathbf{r} s) \rho^{(1)}\left(\mathbf{r}^{\prime} s^{\prime}, \mathbf{r}^{\prime} s^{\prime}\right)}{\left|\mathbf{r}-\mathbf{r}^{\prime}\right|} & d \mathbf{r} d \mathbf{r}^{\prime} \\
= & \frac{1}{2} \int \frac{\rho(\mathbf{r}) \rho\left(\mathbf{r}^{\prime}\right)}{\left|\mathbf{r}-\mathbf{r}^{\prime}\right|} d \mathbf{r} d \mathbf{r}^{\prime}
\end{aligned}
$$

and then the exchange and correlation energies:

$$
\begin{gathered}
\bar{E}_{x}=-\frac{1}{2} \sum_{s} \int \frac{\rho^{(1)}\left(\mathbf{r} s, \mathbf{r}^{\prime} s\right) \rho^{(1)}\left(\mathbf{r}^{\prime} s, \mathbf{r} s\right)}{\left|\mathbf{r}-\mathbf{r}^{\prime}\right|} d \mathbf{r} d \mathbf{r}^{\prime} \\
\bar{E}_{\mathrm{c}}=-\frac{1}{2} \sum_{s s^{\prime}} \int \frac{\chi\left(\mathbf{r} s, \mathbf{r}^{\prime} s^{\prime} ; \mathbf{r s}, \mathbf{r}^{\prime} s^{\prime}\right)}{\left|\mathbf{r}-\mathbf{r}^{\prime}\right|} d \mathbf{r} d \mathbf{r}^{\prime}
\end{gathered}
$$

whose definitions Eqs. (8) and (9) are given respectively in terms of the off-diagonal part of one-electron density matrix $\rho^{(1)}\left(\mathbf{r}^{\prime} s, \mathbf{r s}\right)$ and of the twoelectron density matrix cumulant $\chi\left(\mathbf{r} s, \mathbf{r}^{\prime} s^{\prime} ; \mathbf{r} s, \mathbf{r}^{\prime} s^{\prime}\right)$, the difference between the exact two-electron density matrix and its Hartree-Fock (self-consistent field) estimate.

Although the definition of the Hartree-energy is unique, and constitutes together with the nuclearelectron repulsion energy the part of the total energy that can be written straightforwardly as a simple analytic functional of the one-particle density, the exchange and correlation energies are defined in quantum chemistry and in DFT in different ways. As far as the exchange energy is concerned, one should remark that the one-particle density matrix, appearing in Eq. (8) is supposed to be exact. This quantity is not available even in exact KS theory, where we have at best the one-particle den- sity matrix associated to the single determinant constructed from the exact KS orbitals. By consequence, the exact exchange energy in DFT is in general not equal to $\bar{E}_{x}$.

It must be observed that the usual definition of the correlation energy in quantum chemistry, proposed by Löwdin in Ref. [8] differs from that given in Eq. (9) which follows rather the suggestion due to Kutzelnigg and Mukherjee [9, 10]. This latter definition has the conceptual advantage that it uses the quantities entering Eqs. (7)-(9) irrespective to any approximate method of calculation of the electronic energy. Some authors, (cf. e.g., Refs. [11, 12]) argue that pragmatic DFT methods can be considered as approximations to the two-electron density matrix cumulant.

This situation is as well slightly more complicated in conventional Kohn-Sham theory, where the correlation energy involves also the difference of the exact and KS kinetic energies. However, this kinetic energy contribution can be assimilated to a potential energy term the virtue of the adiabatic connection procedure, which allows one to write the total correlation energy as an average electronelectron interaction over the adiabatic connection path.

\section{Symmetry Nonsensitivity of the Density-only Methods}

\subsection{ARCHETYPICAL EXAMPLE OF EXISTING PROBLEMS}

To better understand the problems which appear in the DFT realm when trying to describe the correct total spin of a many electronic state, we consider the simplest system of two electrons occupying spatial orbitals $|a\rangle$ and $|b\rangle$ (which can be understood as notation for one-dimensional irreducible representations of a point group) and forming corresponding singlet and triplet states ${ }^{1} B$ and ${ }^{3} B$. The relevant wave functions in the coordinate representation are given by:

$$
\begin{aligned}
& \Psi_{1 B}\left(x_{1}, x_{2}\right)= \frac{1}{2}\left(a\left(\mathbf{r}_{1}\right) b\left(\mathbf{r}_{2}\right)+b\left(\mathbf{r}_{1}\right) a\left(\mathbf{r}_{2}\right)\right)\left(\alpha\left(s_{1}\right) \beta\left(s_{2}\right)\right. \\
&\left.-\beta\left(s_{1}\right) \alpha\left(s_{2}\right)\right), \\
& \Psi_{3 B}\left(x_{1}, x_{2}\right)=\frac{1}{2}\left(a\left(\mathbf{r}_{1}\right) b\left(\mathbf{r}_{2}\right)-b\left(\mathbf{r}_{1}\right) a\left(\mathbf{r}_{2}\right)\right)\left(\alpha\left(s_{1}\right) \beta\left(s_{2}\right)\right. \\
&\left.+\beta\left(s_{1}\right) \alpha\left(s_{2}\right)\right), \quad(10)
\end{aligned}
$$


both having the zero projection of the total spin. Following the definitions of the one-electron density matrices Eq. (1) the states Eq. (10) immediately yield exactly the same one-electron density matrix:

$$
\begin{array}{r}
\rho_{2 s+1 B}^{(1)}\left(x, x^{\prime}\right)=\frac{1}{2}\left(\alpha^{*}(s) \alpha\left(s^{\prime}\right)+\beta^{*}(s) \beta\left(s^{\prime}\right)\right)\left(a^{*}(\mathbf{r}) a\left(\mathbf{r}^{\prime}\right)\right. \\
\left.+b^{*}(\mathbf{r}) b\left(\mathbf{r}^{\prime}\right)\right)
\end{array}
$$

irrespective to the total spin of these states. This result is well known for decades and appears even in textbooks [13]. Obviously the density Eq. (2) which is required by the DFT is as well the same for the two spin states.

The exact two electron density matrices calculated according to their definition Eq. (1) from the wave functions Eq. (10) are, however, different:

$$
\begin{array}{r}
\rho_{1,3 B}^{(2)}\left(x_{1} x_{2}, x^{\prime}{ }_{1} x^{\prime}{ }_{2}\right)=\frac{1}{4}\left(\alpha^{*}\left(s_{1}\right) \beta^{*}\left(s_{2}\right) \mp \beta^{*}\left(s_{1}\right) \alpha^{*}\left(s_{2}\right)\right) \\
\times\left(\alpha\left(s^{\prime}{ }_{1}\right) \beta\left(s^{\prime}{ }_{2}\right) b \beta\left(s^{\prime}{ }_{1}\right) \alpha\left(s_{2}{ }^{\prime}\right)\right) \times\left(a^{*}\left(\mathbf{r}_{1}\right) b^{*}\left(\mathbf{r}_{2}\right)\right. \\
\left. \pm b^{*}\left(\mathbf{r}_{1}\right) a^{*}\left(\mathbf{r}_{2}\right)\right)\left(a\left(\mathbf{r}^{\prime}{ }_{1}\right) b\left(\mathbf{r}_{2}{ }_{2}\right) \pm b\left(\mathbf{r}_{1}{ }_{1}\right) a\left(\mathbf{r}^{\prime}{ }_{2}\right)\right)
\end{array}
$$

with the upper sign corresponding to $S=0$ and the lower one to $S=1$. Comparing the above expression with the decomposition Eq. (5), one easily sees that only the cumulant of the two-electron density matrix can be responsible for the distinguishing of the two-electron density matrices for the singlet and triplet states.

The "oversymmetry" of the density (and even of the first order density matrix) with respect to the total spin exemplified by Eq. (11) is not accidental, but is a consequence of a very general result (see Refs. $[14,15]$ and references therein). Even a higher symmetry can be proven [16]. In its modern formulation (Theorem 1 of Ref. [14]) it reads: "The electron density of an arbitrary $\mathrm{N}$-electron system, characterized by the $N$-electron wave function corresponding to the total spin $S$, and constructed on some orthonormal orbital set, does not depend upon the total spin $S$ of the state and always preserves the same form as it is for a single-determinant wave function." The proof given in Ref. [14] relies not upon the spin properties themselves rather on the manifestation of permutation symmetry of the exact wave function in terms of the total spin. We address this issue later in Section 4.2.1.

\subsection{METHODS PROPOSED TO TREAT COINCIDING DENSITIES}

\subsubsection{Multiplet Sum Method}

The first attempt to get around this problem of coinciding densities in the DFT context dates back to the work Ref. [17]. The analysis of problems performed there is precisely repeated in the above two-electron two-orbital model. The prescription Ref. [17] concerning the way out reads as follows: to evaluate correctly the energy of the singlet and triplet states ${ }^{1} B$ and ${ }^{3} B$ in terms of the quantities which can be obtained with use of single determinant wave functions. To do so, one has to address the single determinant function $|a \alpha b \beta|$ which is not a pure spin state, but in fact is a linear combination of two above spin states:

$$
|a \alpha b \beta|=\frac{1}{\sqrt{2}}\left(\left|{ }^{1} B, S_{z}=0\right\rangle+\left|{ }^{3} B, S_{z}=0\right\rangle\right)
$$

Averaging the Hamiltonian over the linear combination Eq. (12) of the pure spin states immediately yields:

$$
\frac{1}{2} E\left({ }^{1} B\right)+\frac{1}{2} E\left({ }^{3} B\right)
$$

The energy of the triplet state entering the above combination can be independently extracted from another single determinant wave function: $|a \alpha b \alpha|$ corresponding to the component of the triplet with the spin projection +1 . Thus one can express the energy of the (nonsingle-determinant) singlet state linearly combining the averages of the Hamiltonian over the single determinant states of which, however, one belongs to the spin projection +1 . Obviously the above move was only possible because the off-diagonal matrix element of the Hamiltonian between the singlet and triplet contributions to the determinant of interest vanishes due to the spin symmetry. The different expressions for $E\left({ }^{1} B\right)$ and $E\left({ }^{3} B\right)$ thus obtained are then treated as required distinct energy functionals to be used to calculate the energy, respectively, for the singlet and triplet states possessing the same one-electron density. It is instructive to check (and in this simple case it can be done by direct evaluation) where the difference between the energy expressions comes from. Inserting the one-electron density matrix Eq. (11) which is the same for both spin states in the definitions of the Hartree and exchange energies Eqs. (7) and (8) 
yields for the both spin states equal Hartree and exchange contributions:

$$
\begin{gathered}
\text { Hartree } \frac{1}{2}[(a a \mid a a)+(a a \mid b b)+(b b \mid a a)+(b b \mid b b)] ; \\
\text { exchange } \frac{1}{2}[(a a \mid a a)+(a b \mid b a)+(b a \mid a b)+(b b \mid b b)] .
\end{gathered}
$$

One can see that (i) the self interaction terms in the Hartree contribution are precisely cancelled by the corresponding terms in the exchange part; (ii) at the same time, obviously, there is no other source where the difference between the spin state energies could come from except the cumulant of the two-electron density matrix and thus the correlation energy as defined by Eq. (9) is responsible for the difference in the resulting expressions:

$$
\begin{aligned}
& E\left({ }^{1} B\right)=(a a \mid b b)+(a b \mid b a) \\
& E\left({ }^{3} B\right)=(a a \mid b b)-(a b \mid b a)
\end{aligned}
$$

On the other hand one may notice that the classification of the energy contributions as exchange or correlation ones by Eqs. (8) and (9) is in some way arbitrary as well. Indeed, for the above model the energy of the triplet state with the spin projection +1 is exactly the sum of the Hartree and exchange contributions because the latter state is represented by a single determinant wave function for which the cumulant precisely vanishes. However, the equal energy for the triplet state with the zero spin projection breaks down differently: into the Hartree, exchange, and correlation contributions, where the Hartree contribution is the same as in the case of the spin projection +1 , but only the sum of the exchange and correlation contributions is the same for different values of $S_{z}$.

The above way leading to the energy expressions for different spin states is not completely satisfactory (and not clearly generalizable): although formally, the results can be treated as functionals of the density the difference of the two energy expressions is obtained by a kind of trick. Referring to the triplet component with $S_{z}=+1$ in the derivation of the multiplet energy looks out as an alien element (in fact the energy is uniquely determined by the spatial multipliers in the wave functions Eq. (10), without any reference to the spin components at all). This strange element of the derivation ap- peared in order to compensate somehow the element of the general theory which is missing in the DFT, the cumulant of the two-electron density matrix. Despite this criticism, the result of the derivation is very transparent; it reduces to deriving according to McWeeny's notice in Ref. [18] "of a particular type of energy expression-irrespective of the nature of the wavefunction," namely onelinear in the Coulomb and exchange two-electron integrals over the involved orbitals.

Further development of this approach is based on the assumption that it is always (or at least for unspecifically wide class of cases) possible to express the energy of a pure spin multiplet state allowable for a given number of electrons/orbitals as a linear combination:

$$
E(n \Gamma S)=\sum_{i} w_{i}^{n \Gamma S} E_{i}
$$

where $E_{i}$ are the diagonal matrix elements of the energy operator taken with respect to all necessary Slater determinants (numbered by $i$ ). The one-electron density (matrices) corresponding to these determinants are different and the whole scheme becomes workable provided the set of the coefficients (weights) $w_{i}^{\Gamma s}$ exists and they are uniquely defined by the spin and symmetry quantum numbers $\Gamma$ and $S$ and other quantum numbers $n$ serving to distinguish potentially existing states with equal $\Gamma$ and $S$. Apparently, only a restricted number of examples of such functional forms is known. The reason is quite simple and the above consideration allows to single out the range of cases where the derivation analogous to that of Eq. (12) can be performed. It applies if the total spin allows to completely distinguish the electronic states. In this case, the energy of the state of the highest available spin $S_{\max }$ can be expressed through a single determinant function (the cumulant is vanishing) with the highest available projection of the total spin. Then this result can be used to evaluate the energy of the state with $S_{\max }-1$, etc. The recipe immediately fails as soon as multiple states of the same total spin appear in the system. This is, however, the everyday life, so in what follows we switch to considering further possibilities of constructing the energy functionals useful in this situation.

\subsubsection{Restricted Open Shell KS (ROKS) Method}

The situation with reproducing total spin dependence of the energy as it appears in the DFT context 
is by no means unique: the same problem arises in the Hartree-Fock-Roothaan (HFR) context because the latter lacks any adequate representation of the cumulant of the two-electron density matrix as well. Within the "extended" HFR context, some ways out have been proposed. Incidentally, the method of Ref. [17] is precisely the Slater multiplet sum method Ref. [19] which migrated from the HFR to the DFT context. Another option is the ROHF (restricted open shell Hartree-Fock) method whose respective migration resulted in a range of the ROKS (restricted open shell Kohn-Sham procedures [20,21]) being the DFT counterpart of the former. Despite different appearance they have many common features (and we do not address here the methods based on the statistical-ensembleaveraging).

The derivation of the ROHF (or equivalently "old MC SCF," see below) bases on the general expression of the form:

$$
E(n \Gamma S)=\sum_{i j} C_{i}^{n \Gamma S} C_{j}^{n \Gamma S} H_{i j}
$$

where $C_{i}^{n \Gamma S}$ are the expansion coefficients of the eigenfunction $\Psi_{n \Gamma S}\left(x_{1}, x_{2}, x_{3}, \ldots x_{N}\right)$ of the many-electron hamiltonian over some appropriate basis states $\Phi_{i}\left(x_{1}, x_{2}, x_{3}, \ldots x_{N}\right)$ (e.g., the Slater determinants, see, however, below). In this case, no alien states of wrong spin projection may appear. On the other hand, the contribution of the off-diagonal elements $H_{i j}$ to the energy may be nontrivial (in contrast with Eq. (16)). The knowledge of the expansion coefficients $C_{i}^{n \Gamma S}$ in general requires diagonalization of the Hamiltonian matrix making the expansion coefficients and thus the energy itself some sophisticated irrational function of the Hamiltonian matrix elements including two-electron integrals. It was Roothaan [22] who first noticed that certain states $\Psi_{n \Gamma s}$ of atoms and linear molecules, even those requiring many-determinant (multireference, multiconfigurational) wave functions, yield energy expressions which are linear with respect to twoelectron integrals (ii $\mid j j$ ) and (ij| ji) (respectively, Coulomb and exchange ones). It is only possible if the wave function expansion coefficients $C_{i}^{n \Gamma S}$ in Eq. (17) can be determined on the symmetry grounds, that is, without nontrivial diagonalization. In this case, there is no need that the off-diagonal elements $H_{i j}$ which are linear expressions in the two-electron integrals and thus give a linear contribution to the energy functional disappear as required by Eq. (16).
Only the possibility to have the expansion coefficients $C_{i}^{n \Gamma S}$ independent on the specific values of the Hamiltonian matrix elements is the true prerequisite for obtaining the expressions for the energy of the required (linear) form. Nevertheless, the number of cases when the described procedure was possible in fact reduces to the $p^{n}$ states of atoms and $\pi^{n}$ and $\delta^{n}$ states of linear molecules. Similarly, the ROKS scheme proposed in Ref [21] and representing a migration of the Roothaan's reasoning to the DFT context allowed to obtain the functional forms for the same set of states: $p^{n}, \pi^{n}$, and $\delta^{n}$. Thus the forecast of the year 1960 due to Roothaan [22]: "It is a relatively simple matter to extend the open-shell theory just presented in such a way that other important classes of atomic states can be accommodated, as for instance, the $d^{N}$ configurations for the transition elements. We postpone such generalizations for the present, and include whatever new treatments may be necessary with the actual applications planned for the future" never became true and the $d^{N}$ states generally cannot be squeezed in the ROHF/ROKS scheme.

Under other angle of view, validity of the Roothaan or similar schemes means that the cumulant of the two-electron density matrix can be in some particular case recovered by symmetry based manipulations. In the cases considered by Roothaan himself and recently used in the DFT context in Ref [21], the possibility of obtaining closed expressions for the energy functional in terms of two-electron integrals over orbitals involved is stipulated by additional symmetry of the system (in the chemical context it goes about additional symmetry group $G$ with irreducible representations $\Gamma$, where $G=$ $S O(3)$ for an atom $G=S O(2)$ for a linear molecule, and may be some point group for other molecules) which allows to figure out the expansion coefficients $C_{i}^{n \Gamma S}$. It is clear that for an overwhelming majority of cases, it is impossible to find any nontrivial symmetry group $G \neq C_{1}$ which predefines a restricted character of any Roothaan-like treatment. It is thus our next purpose is to explore other possibilities of designing energy functionals distinguishing the states of different spin multiplicities in the DFT context.

\section{Spin and Unitary Symmetry of the Electronic Wave Function}

As we mentioned previously any reference to the spin projections throughout the derivation of the 
energy expressions for the two-electron two-orbital model looks out as an alien element. The ultimate reason for that is that the nonrelativistic Hamiltonian does not depend on spin variables at all and the energy itself as well as the differences in its form for different spin states originates solely from the spatial multiplier of the many-electronic wave function (spatial function). The idea to restrict the entire consideration by those spatial functions persists almost from the beginning of the quantum chemistry and is known as "spin-free quantum chemistry" [23]. It can be given different formulations of which we use one based on the unitary group (see Ref. [18]). We briefly remind its basic facts in the Appendix.

\subsection{UNITARY SYMMETRY OF THE SPATIAL MULTIPLIER}

The construct using the permutational symmetry of the spatial part of the wave function had been used for developing the so called generalized Hartree-Fock procedure [24] which had numerous descendants (see e.g., [25, 26]). They basically performed the task of presenting the energy in the HFR-like form: linear with respect to Coulomb and exchange integrals over the orbitals involved with the coefficients dependent on the permutational symmetry of the spatial part of the wave function and thus on the total spin. The permutational symmetry, however, addresses the many-electron wave functions in the coordinate representation which is of restricted use in quantum chemistry. By contrast the wave functions actually used are those in the representation of the occupation numbers of the orbitals involved. For that reason, it is more practical to switch to labeling of the many-electron functions by irreducible representations of the unitary group which are closely related to those of the $S_{N}$ group. The corresponding construct is described in the Appendix.

\subsection{PHYSICAL OUANTITIES IN TERMS OF UNITARY GROUP}

Going to the representation of the $U(M)$ group has that advantage that it allows to easily write down the energy of many electron states. This is done as follows: for each Young pattern $Y$ one can construct the set of generators $\mathbf{E}_{i j}^{Y}(i j=1 \div M)$ of the group $U(M)$ acting in the space of the irreducible representation $Y=\Upsilon(M, N, S)$ whose matrix elements between the tableaux $v$ and $v^{\prime}$ can be calculated irrespective to the physical nature of the system described. The set of generators completely defines the action of the group $U(M)$ in the irreducible subspace of its tensors of the rank $N$ with the permutational/spin symmetry stipulated by the Young pattern $Y$.

The diagonal generators $\mathbf{E}_{i i}^{Y}$ are diagonal in the basis of Young tableaux and their matrix elements are equal to the occupation number $\left(n_{i}=2,1,0\right)$ of the $i$-th orbital in the Young tableau Yv:

$$
\left\langle Y v\left|\mathbf{E}_{i i}^{\Upsilon}\right| Y v^{\prime}\right\rangle=\delta_{v v^{\prime}}\left\langle\mathbf{E}_{i i}^{\Upsilon}\right\rangle_{Y v}=\delta_{v v^{\prime}} n_{i}
$$

By contrast off-diagonal generators $\mathbf{E}_{i j}^{\gamma}$ (raising ones if $i>j$ and lowering ones if $j>i$ ) have nonvanishing matrix elements $\left\langle Y_{v}\left|\mathbf{E}_{i j}^{Y}\right| Y_{v^{\prime}}\right\rangle$ if the tableau $v^{\prime}$ contains at least one orbital symbol $j$, whereas the tableau $v$ contains one less orbital symbol $j$ than $v^{\prime}$ and one more orbital symbol $i$ than it. From this, it follows that the off-diagonal generators $\mathbf{E}_{i j}^{Y}(i \neq j)$ have no nonvanishing diagonal matrix elements.

\subsubsection{One-Electron Density in the Unitary Group Formalism}

The generators $\mathbf{E}_{i j}^{Y}$ are by definition the components of the spatial one-electron density operator restricted to the subspace of the states belonging to the $Y$ pattern (those having transformation properties of the corresponding rank $N$ tensors with the permutational symmetry stipulated by the Young pattern $Y$ or equivalently having the total spin prescribed by this pattern):

$$
\mathbf{E}_{i j}^{\Upsilon}=\sum_{\sigma} P^{\Upsilon} \psi^{+}(i \sigma) \psi(j \sigma) P^{\Upsilon},
$$

where $P^{\curlyvee}$ stands for the operator projecting $N$-electron wave function to the subspace of the functions with the spatial part having the permutational symmetry of the irreducible representation $Y$ of either $S_{N}$ or $U(M)$ groups. It is remarkable to note that the Young tableaux states have an important property similar to that of the Slater determinants: the oneelectron density matrices generated from such states are diagonal in the basis of the orbitals involved in their construction.

With the use of this construct, one can easily check the validity of the Kaplan's Theorem 1. Indeed, for whatever Young tableau Yv, the one electron density pertinent to the corresponding $\mathrm{N}$-electron state reads: 


$$
\begin{aligned}
\rho_{Y v}\left(\mathbf{r}, \mathbf{r}^{\prime}\right)= & \sum_{s} \rho_{Y v}^{(1)}\left(\mathbf{r} s, \mathbf{r}^{\prime} s\right) \\
= & \sum_{s}\left\langle Y v\left|\psi^{+}(\mathbf{r} s) \psi\left(\mathbf{r}^{\prime} s\right)\right| Y v\right\rangle \\
= & \sum_{\sigma} \sum_{s} \sigma^{*}(s) \sigma(s) \sum_{i j} \varphi_{i}^{*}(\mathbf{r}) \varphi_{j}\left(\mathbf{r}^{\prime}\right) \\
& \times\left\langle Y v^{+}(i \sigma) \psi(j \sigma) \mid Y v\right\rangle= \\
= & \sum_{i j} \varphi_{i}^{*}(\mathbf{r}) \varphi_{j}\left(\mathbf{r}^{\prime}\right)\left\langle Y v\left|\mathbf{E}_{i j}^{\Upsilon}\right| Y v\right\rangle= \\
= & \sum_{i j} \varphi_{i}^{*}(\mathbf{r}) \varphi_{j}\left(\mathbf{r}^{\prime}\right) \delta_{i j}\left\langle Y v\left|\mathbf{E}_{i i}^{Y}\right| Y v\right\rangle \\
= & \sum_{i} n_{i} \varphi_{i}^{*}(\mathbf{r}) \varphi_{i}\left(\mathbf{r}^{\prime}\right)
\end{aligned}
$$

which in turn does not depend on the permutation symmetry labels $Y v$, which is the only connection to the total spin. Thus even the spatial density matrix (not only the density) is permutation/spin independent as stated in Ref. [14].

\subsubsection{Energy in the Unitary Group Formalism}

Further development is based on the possibility to express the blocks of the Hamiltonian matrix pertaining to $N$ electrons in $M$ orbitals with total spin $S$ through the generators $\mathrm{E}_{i j}^{\mathrm{Y}}$, with $Y=Y(M, N$, $S)$. The required representation reads Ref. [18]:

$$
\begin{gathered}
\mathbf{H}=\oplus_{\Upsilon} \mathbf{H}^{\Upsilon} \\
\mathbf{H}^{\Upsilon}=\sum_{i j} h_{i j} \mathbf{E}_{i j}^{\Upsilon}+\frac{1}{2} \sum_{i j k l}(i j \mid k l)\left(\mathbf{E}_{i j}^{\Upsilon} \mathbf{E}_{k l}^{\Upsilon}-\delta_{j k} \mathbf{E}_{i l}^{\Upsilon}\right)
\end{gathered}
$$

The matrix elements $h_{i j}$ are the sums of the respective matrix elements of the kinetic energy $\hat{T}_{e}$ of electrons and of the external Coulomb potential $\hat{V}_{n e}$; the quantities $(i j \mid k l)$, the two-electron matrix elements of the Coulomb interactions.

For each of the states $\left|Y_{v}\right\rangle$ represented by the Young tableau with the Young pattern $Y$ and the filling $v$ (this information suffice to define the spatial part of the $N$-electron wave function) the expectation value of the energy reads:

$$
E(\Upsilon v)=\sum_{i j} h_{i j}\left\langle\mathbf{E}_{i j}^{\Upsilon}\right\rangle_{\Upsilon v}+\frac{1}{2} \sum_{i j k l}(i j \mid k l)\left\langle\left(\mathbf{E}_{i j}^{\Upsilon} \mathbf{E}_{k l}^{\Upsilon}-\delta_{j k} \mathbf{E}_{i l}^{\Upsilon}\right)\right\rangle_{\Upsilon v}
$$

For the one-electron contribution to the energy one gets:

$$
\sum_{i j} h_{i j}\left\langle\mathbf{E}_{i j}^{\Upsilon}\right\rangle_{\Upsilon v}=\sum_{i} h_{i i}\left\langle\mathbf{E}_{i i}^{\Upsilon}\right\rangle_{\Upsilon v}=\sum_{i} h_{i i} n_{i}
$$

and the Coulomb interaction of electrons is expressed through the Coulomb and exchange integrals with respect to the orbitals involved in the construction of the states represented by the Young tableaux:

$$
\begin{aligned}
\text { Hartree } & \frac{1}{2} \sum_{i j}(i i \mid j j)\left\langle\mathbf{E}_{i i}^{\Upsilon} \mathbf{E}_{j j}^{\Upsilon}\right\rangle_{\Upsilon_{v}}+\text { exchange + correlation } \\
\times & \frac{1}{2} \sum_{i \neq j}(i j \mid j i)\left\langle\mathbf{E}_{i j}^{\Upsilon} \mathbf{E}_{j i}^{\Upsilon}-\mathbf{E}_{i i}^{\Upsilon}\right\rangle_{\Upsilon_{v}}-\sum_{i}(i i \mid i i)\left\langle\mathbf{E}_{i i}^{\Upsilon}\right\rangle_{Y_{v}}
\end{aligned}
$$

The Young tableau states $Y v$ are the eigenstates of the diagonal generators $\mathbf{E}_{i i}^{Y}$. For that reason the Hartree contribution to the energy can be rewritten:

$$
\operatorname{Hartree} \frac{1}{2} \sum_{i j}(i i \mid j j)\left\langle\mathbf{E}_{i i}^{\Upsilon}\right\rangle_{\Upsilon_{v}}\left\langle\mathbf{E}_{j j}^{\Upsilon}\right\rangle_{\Upsilon_{v}}=\frac{1}{2} \sum_{i j}(i i \mid j j) n_{i} n_{j}
$$

in terms of the products of the one-electron densities. From this, we see that the Hartree part of the Coulomb energy is uniquely defined by the occupation numbers of the spatial orbitals, that is, only by the spatial density in the representation of orbitals. We see that as in the other representations the Hartree term is contaminated by the self-interaction of electrons and that the principal effect for which the true exchange term is responsible in the HFR context, the avoiding of the self interaction, is guaranteed by the specific form of the coefficient at the integrals of the (iilii) type which must be absorbed by the exchange contribution to the energy. The averages of the off-diagonal generators' products entering the expression Eq. (22) are not, however, uniquely defined either by the occupation numbers of the orbitals in the tableau $Y v$ or by the total spin, prescribed by the pattern $Y$. They depend also on the mutual positions of the orbital symbols in the tableau. This is precisely the result obtained many years ago in Ref. [27] under the name of the spinfree self consistent field theory.

From the ROHF (old MCSCF) point of view the result Eqs. (21) and (22) can be considered as a recipe of obtaining the coupling coefficients $a_{i j}$ and $b_{i j}$ at the Coulomb and exchange integrals in the 
ROHF expressions for the energy, which incidentally acquire the $Y v$ dependence:

$$
\begin{gathered}
a_{i j}^{Y v}=\left\langle\mathbf{E}_{i i}^{\Upsilon} \mathbf{E}_{j j}^{Y}-\delta_{i j} \mathbf{E}_{i i}^{\Upsilon}\right\rangle_{Y v} \\
b_{i j}^{Y v}=\left\langle\mathbf{E}_{i j}^{\Upsilon} \mathbf{E}_{j i}^{\Upsilon}-\mathbf{E}_{i i}^{\Upsilon}\right\rangle_{Y v}
\end{gathered}
$$

Turning back to expressions Eq. (17), one can say that constructing the spatial Young tableaux states provide the expansion coefficients $C_{i}^{\gamma v}$ for the respective linear combinations of the $\mathrm{N}$-electron Slater determinants, yielding the total spin specified by the Young pattern. These coefficients are derived by purely symmetry reasons and do not depend on the matrix elements of the Hamiltonian thus satisfying the requirement of "universality." On the other hand it is obvious that specifying the total spin only does not suffice to specify the electronic state. The procedure implied by Eq. (21) provides for each allowable set of $M, N, S$ the whole bunch of energy expressions labeled by the rows $v$ of the irreducible representation $Y=Y(M, N, S)$.

\subsection{MULTIPLET SUM METHOD FROM THE UNITARY PERSPECTIVE}

The first usage of the above formalism is to repeat the success of the MSM in case of two electrons in two orbitals without addressing explicitly the foreign component of the triplet state with $S_{z}=+1$. Indeed, the spatial parts of the multiplet states in Eq. (10) are equivalently represented as the Young tableaux states:

$$
\begin{gathered}
{ }^{1} B|\underline{\mathrm{a} \mid \mathrm{b}}\rangle \\
{ }^{3} B\left|\frac{a}{b}\right\rangle
\end{gathered}
$$

Two electrons in two orbitals form only one spatial function for the spin triplet state, but in addition to one given above two more functions compatible with the spin singlet state:

$$
|\mathrm{a}| \mathrm{a}\rangle, \quad|\mathrm{b}| \mathrm{b}\rangle
$$

are available. Three spatial functions compatible with the spin singlet state together form a basis of the three-dimensional irreducible representation of the group $U$ (2) corresponding to the total spin 0 . The single spatial function compatible with the spin triplet state spans the one-dimensional irreducible representation of the group $U(2)$. The Young pattern label $Y$ here can be replaced by indicating the total spin only. Then the generator $\mathbf{E}_{a b}^{S=1}=0$, but for $S=0$ one has:

$$
\left\langle\mathrm{a} \mathbf{a}\left|\mathbf{E}_{a b}^{S=0}\right| \mathrm{a} \mathrm{b}\right\rangle=\sqrt{2}=\left\langle\mathrm{b} \mathbf{b}\left|\mathbf{E}_{a b}^{S=0}\right| \mathrm{a} \mathbf{b}\right\rangle
$$

These values suffice to perform the matrix multiplication of the generators $\mathbf{E}_{a b}^{S=0} \mathbf{E}_{b a}^{S=0}$ in the general expressions Eq. (22), so that we obtain for the contribution of the average interaction to the energy:

$$
\begin{aligned}
\mathbf{E}\left({ }^{1} B\right) & =(a a \mid b b)+(a b \mid b a) \\
\mathbf{E}\left({ }^{3} B\right) & =(a a \mid b b)-(a b \mid b a)
\end{aligned}
$$

as it should be. We see that the archetypical result is reproduced within the Young tableaux technique without addressing the component of the spin multiplet with a foreign value of the spin projection. Also the self-interaction contamination is removed automatically.

\subsection{DFT IMPLICATIONS}

All the above treatment was not in any way related to the DFT realm. The possibility of establishing such a relation can be based on the recognition of the fact that the symmetry (in particular the total spin) dependence must be extraneously introduced into DFT considerations [28, 29] analogously to the treatment by Filatov and Shaik Ref. [21]. The unitary group formalism allows us to conclude that for a given set of consistent values of $M, N, S$ one arrives to the family of functionals labeled by the rows $v$ of the irreducible representation $Y=Y(M, N, S)$ of the $U(M)$ group. The spin symmetry features of these functionals are condensed in the $\left\langle\mathbf{E}_{i j}^{Y} \mathbf{E}_{j i}^{Y}-\mathbf{E}_{i i}^{\Upsilon}\right\rangle_{Y}\left(\right.$ or $\left.a_{i j}^{Y v}, b_{i j}^{Y v}\right)$ coefficients given above.

The energy matrix elements reflecting specific features of the system can be easily figured out. The coefficients $a_{i j}^{Y v}$ for the Coulomb integrals (ii $j j$ ) which define the Hartree part of the Coulomb energy are known, but they are of no practical use in the DFT context, where the Hartree part of the interaction is determined directly from the electron density. Relatively, problematic (in the DFT context) is to decide where the energy matrix element to be combined with the coupling coefficients $\left\langle\mathbf{E}_{i j}^{\gamma} \mathbf{E}_{j i}^{\gamma}-\mathbf{E}_{i i}^{\gamma}\right\rangle_{Y v}$ (exchange) and - $\left\langle\mathbf{E}_{i i}^{Y}\right\rangle_{Y v}$ (self-inter- 
action) has to come from. This choice must be compatible with various theoretical settings. First of all we notice that if a hybrid functional is used which contains some fraction of the Hartree-Fock exchange the latter must be modified accordingly so that the corresponding (ij|ji) integrals over the Kohn-Sham orbitals be included with the correct coefficients $\left\langle\mathbf{E}_{i i}^{\gamma} \mathbf{E}_{j i}^{\gamma}-\mathbf{E}_{i i}^{\gamma}\right\rangle_{Y v}$. The same applies to the integrals (ii|ii) which together with coefficients $-\left\langle\mathbf{E}_{i i}^{\gamma}\right\rangle_{Y_{v}}$ will take care about some fraction of selfinteraction.

Further concerns are related with the treatment of the nontrivial parts of the exchange-correlation functionals within the $Y v$ numbering of the spin (permutation) states. This can be solved on the basis of certain consistency requirements. Among possible consistency requirements, the most natural is that with the TDDFT. The TDDFT approximation is equivalent to constructing the corresponding time evolution of the many-electronic state in the basis of single electron excitations (particle-hole pairs) above the KS single determinant wave function. Leaving aside the question of the area of applicability of such an approach, we notice that it requires an estimate of the twoelectron integrals coupling between different singly excited determinants. The interaction appears as second functional derivative of the energy with respect to density (first functional derivative of the exchangecorrelation functional). In the orbital representation, these derivatives acquire the necessary form of twoelectron integrals with the kernels determined by the form of the used exchange-correlation functional. On the other hand the (ijji), integrals appear in ROHF and in unitary group formalism for $Y_{v}$ states as a consequence of configuration interaction between different Slater determinants implicitly entering in the expansion of the Young tableau state $Y v$. Thus to ensure the compatibility of the corresponding components of the theory, the couplings between the elementary excitations in TD-DFT and between Slater determinants in expansions of $Y v$ states must be the same. Thus they can be expressed through the integral kernels of the interaction (e.g., according to [30]):

$$
(i j \mid j i)^{x c}=\iint \varphi_{i}^{*}(\mathbf{r}) \varphi_{j}(\mathbf{r}) f^{x c}\left(\mathbf{r}, \mathbf{r}^{\prime}\right) \varphi_{j}^{*}\left(\mathbf{r}^{\prime}\right) \varphi_{i}\left(\mathbf{r}^{\prime}\right) d \mathbf{r} d \mathbf{r}^{\prime} ;
$$

where

$$
f^{x c}\left(\mathbf{r}, \mathbf{r}^{\prime}\right)=\frac{\delta v^{x c}(\mathbf{r})}{\delta \rho\left(\mathbf{r}^{\prime}\right)}
$$

Namely these quantities must be inserted in the expressions for the exchange-correlation energies to get these later consistent with the total spin/permutation symmetry of the underlying many-electronic ground state. This is also in agreement with the way of constructing the coupling operators by Filatov and Shaik in their version of ROKS Ref. [21] and a similar procedure can be easily designed for the $Y v$ labeled states.

\subsection{FURTHER EXAMPLES}

As we mentioned many times, the spin in general does not suffice to distinguish many electronic states with the same one-electron density, which produces problems in describing corresponding states in the DFT. The only example of the usage of the unitary group formalism given so far was, however, the simplest case when the total spin labeling was sufficient. Below we briefly exemplify the features one should expect in general case when there exist Young tableaux differing by the positions of the orbital symbols in these tableaux. In this case, one can say that for given $M, N, S$ uniquely defining the irreducible representation $Y$ of the group $U(M)$ and for the row $v$ of the latter defined by a specific location of the orbital symbols in the tableau a "Hartree-Fock-like" energy functional can be written whose electron-electron interaction part is given by Eq. (22). It can be optimized with respect to the expansion coefficients of the involved orbitals over the AO's basis yielding an effective Fockian matrix whose eigenvectors are precisely the orbitals involved in the construction of the Young tableau state in the same manner as it is in the ROHF/ ROKS.

It is easy to check that the positions of the orbital indices in the tableaux really matter. For example, for two Young tableaux states:

$$
|Y v\rangle=\left|\begin{array}{|l|l}
\mathrm{a} & \mathrm{b} \\
\hline \mathrm{c} & \mathrm{d}
\end{array}\right\rangle ;\left|Y v^{\prime}\right\rangle=\left|\begin{array}{|l|l}
\mathrm{a} & \mathrm{c} \\
\hline \mathrm{b} & \mathrm{d}
\end{array}\right\rangle
$$

both representing singlet states of four electrons in four orbitals with equal one-electron density matrices, the contributions to the energy functionals of the form Eq. (22), proportional to the exchange integrals, respectively, are [31]:

$$
\begin{aligned}
|Y v\rangle:(a b \mid b a)+(c d \mid d c)-\frac{1}{2}[(a c \mid c a)+(a d \mid d a) & \\
& +(b c \mid c b)+(b d \mid d b)]
\end{aligned}
$$




$$
\begin{array}{r}
\left|\Upsilon v^{\prime}\right\rangle:-(a b \mid b a)-(c d \mid d c)+\frac{1}{2}[(a c \mid c a)+(a d \mid d a) \\
+(b c \mid c b)+(b d \mid d b)]
\end{array}
$$

where the Hartree and the self-interaction correcting terms are omitted for brevity.

Remarkably enough neither of the expressions Eq. (28) (combined with other necessary temrs) yields a lower energy a priori, which one is lower depends on the relations between the molecular integrals involved. At this point one can return to the qualitative interpretation of the Young tableaux with different positions of the orbital symbols as of reflecting different "pairing schemes." Indeed, the states in Eq. (27) can be, respectively, treated (and this is in accord with the energy expressions Eq. (28)) as pairwisely coupling electrons in the states $a$ and $b$ and $c$ and $d$ to the singlets and triplets, then coupling these intermediate states to the final singlet states.

On the other hand, one can easily conclude that for the above pair of Young tableaux $Y v$ and $Y v^{\prime}$ for which $n_{i}=n_{j}=1$ and the difference between them is only the positions of the orbital symbols $b$ and $c$ in the tableaux Eq. (27), the operators $\mathbf{E}_{i j}^{\gamma} \mathbf{E}_{j i}^{\Upsilon}$ entering as multipliers of the (ij| ji) exchange integrals in the exact Hamiltonian yield also an off-diagonal matrix element of the Hamiltonian [31]:

$$
\begin{array}{r}
\left\langle Y v\left|\mathbf{H}^{Y}\right| Y v^{\prime}\right\rangle=-\frac{\sqrt{3}}{2}((a c \mid c a)-(a d \mid d a)-(b c \mid c b) \\
+(b d \mid d b)) \neq 0,
\end{array}
$$

which shows that in this case a $2 \times 2$ diagonalization is required for obtaining the electronic energy exactly. Thus the energy functional becomes a square root irrational function of the two-electron integrals rather than a linear one. Additional symmetry relations may produce the energy expression linear in the Coulomb and the exchange integrals (in the above example it suffice that exchange integrals $(a c \mid c a),(a d \mid d a)$ and $(b c \mid c b),(b d \mid d b)$ are pairwisely equal (which makes Eq. (29) be zero) or alternatively that the exchange integrals satisfy the equality:

$$
\begin{array}{r}
(a b \mid b a)+(c d \mid d c)=\frac{1}{2}[(a c \mid c a)+(a d \mid d a)+(b c \mid c b) \\
+(b d \mid d b)]
\end{array}
$$

which makes the diagonal matrix elements of the Hamiltonian be equal for the states represented by Eq. (27). Both symmetries yield specific forms of the $2 \times 2$ configuration interaction matrix and by this allow the diagonalization to be feasible on the purely symmetry grounds.

\section{State-Specific \\ Exchange-Correlation Functionals for Atomic d-Shells}

The above notion of irrationality shows that even the unitary group formalism does not solve the problem of constructing density functionals for the specific correlated states. Although the unitary group formalism allows to significantly contract the expansions of the states of the definite total spin (in fact the $Y v$ labeled states become single-configuration, albeit each of them is a combination of many Slater determinants) the nonlinearity of the energy expression with respect to the two-electron integrals hinders constructing the symmetry adapted functionals along the lines suggested above. This problem manifests itself in the description of the many-electronic states in the $d$-shells of transition metal ions. Using the unitary group formalism also in this case does not allow to go further than the Roothaan old MC SCF theory as described in Ref. [22]. There the spin/angular momentum dependent coupling coefficients $a_{i j}$ and $b_{i j}$ had been introduced ultimately to express the cumulant of the two-electron density matrix using symmetry considerations. They are valid only if the multiplet states can be uniquely obtained by applying operators projecting the Young tableau states to the specific rows of the irreducible representations of the $S O(3)$ or $S O(2)$ groups (atoms and linear molecules, respectively). In these two cases, moderately simple expressions for the classifying operators (respectively, $L^{2}$ and $L_{z}$ ) in terms of the generators $\mathbf{E}_{i j}^{Y}$ can be written and used for constructing the required symmetry adapted combinations of the Young tableau states. In the case of the atomic $p$-shells (and molecular $\pi$ - and $\delta$-shells), the number of the $S O(3)(S O(2))$ symmetry labels (different values of the orbital momentum $L$ ) produced by the projection of the Young tableau states to the definite $L^{2}$ states suffice to distinguish all different energies in these shells. In the case of atomic $p^{n}$-states the symmetry $S O(3)$ reduces also the number of independent two-electron integrals (including both the 
TABLE I

The energy expressions of the many-electron states in the $d$-shell of the $\mathrm{Fe}^{2+}$ ion.

\begin{tabular}{lccc}
\hline$E\left({ }_{ \pm}^{1} S\right)=E_{0}$ & $+10 F_{2}$ & $+6 F_{4}$ & $\pm \frac{1}{2} \sqrt{3,088 F_{2}^{2}-26,400 F_{2} F_{4}+13,3200 F_{4}^{2}}$ \\
$E\left({ }_{ \pm}^{1} D\right)=E_{0}$ & $+9 F_{2}$ & $-76.5 F_{4}$ & $\pm \frac{1}{2} \sqrt{1,296 F_{2}^{2}-10,440 F_{2} F_{4}+30,825 F_{4}^{2}}$ \\
$E\left({ }^{2} F\right)=E_{0}$ & & $+48 F_{4}$ & 1 \\
$E\left({ }_{ \pm}^{1} G\right)=E_{0}$ & $-5 F_{2}$ & $-6.5 F_{4}$ & $\pm \frac{1}{2} \sqrt{708 F_{2}^{2}-7,500 F_{2} F_{4}+30,825 F_{4}^{2}}$ \\
$E\left({ }^{1} I\right)=E_{0}$ & $-15 F_{2}$ & $-9 F_{4}$ & 1 \\
$E\left({ }_{ \pm}^{3} P\right)=E_{0}$ & $-5 F_{2}$ & $-76.5 F_{4}$ & $\pm \frac{1}{2} \sqrt{912 F_{2}^{2}-9,960 F_{2} F_{4}+38,025 F_{4}^{2}}$ \\
$E\left({ }^{3} D\right)=E_{0}$ & $-5 F_{2}$ & $-129 F_{4}$ & 1 \\
$E\left({ }_{ \pm}^{3} F\right)=E_{0}$ & $-5 F_{2}$ & $-76.5 F_{4}$ & \\
$E\left({ }^{3} G\right)=E_{0}$ & $-12 F_{2}$ & $-94 F_{4}$ & \\
$E\left({ }^{3} H\right)=E_{0}$ & $-17 F_{2}$ & $-69 F_{4}$ & \\
$E\left({ }^{5} D\right)=E_{0}$ & $-21 F_{2}$ & $-189 F_{4}$ & \\
\hline
\end{tabular}

$E_{0}$ is given by the expression: $E_{0}=n_{d} T+n_{d}\left(n_{d}-1\right) A / 2$ where $T$ is the kinetic energy per electron, $A=F_{0}-49 F_{4}$ and $n_{d}$ is the number of electrons in the $d$-shell.

Coulomb and exchange ones) to only two independent Slater-Condon parameters $F_{k}(p p) ; k=0,2$. This allowed the authors of Ref. [32,33] to develop state-specific functionals for the atomic $p^{n}$-states. It turns out, however, that for the $d$-shells it does not suffice for a major part of the atomic electronic terms of the transition metal ions [34]. Even in free ions where the multiple terms having the same spin and orbital momentum do exist and their energies cannot be expressed linearly through the two-electron integrals. Despite the high-symmetry situation of a free atom (ion) which reduces all the twoelectron integrals to a limited number (three) Slater-Condon parameters $F_{k} ; k=0,2,4$ in the free ions the energies of the multiplets require $2 \times 2$-diagonalization and thus their analytical expressions contain square roots (for a handy reference see [34]). This moment is crucial, it is not possible to get rid out of the irrationality (square root) in the expression for the energy by linearly combining the parameters of the Hamiltonian.

The situation clearly becomes less favorable in lower symmetries or in larger subshells (e.g., partially filled $f$-shells) where the terms of the same spin and symmetry span the subspaces of dimensionalities higher than two. For example, in the octahedral environment the LS states of $d^{4}-\left(d^{6}-\right)$ configuration span up to seven-dimensional subspaces of many-electronic states [35]. Clearly, at an arbitrarily low symmetry the problem of linearly expressing the exact energy of many-electronic terms through the Coulomb and exchange integrals cannot be solved and obviously the energy of any of such multiple terms cannot be expressed as a linear combination of Coulomb and exchange integrals. In what follows below, we restrict ourselves to the case of atomic $d$ - shells and the square root irrationalities in the state-specific expressions for the energy trying to squeeze the simplest thinkable irrationality reflecting nontrivial correlations in a kind of generalized density functional.

\subsection{THE EXAMPLE OF $\mathrm{Fe}^{2+}$ ION}

We concentrate on the free $\mathrm{Fe}^{2+}\left(d^{6}\right)$ ion which is an important object in the studies of biologically active transition metal complexes and following [34] provides a rich system of nontrivially correlated multiple states in its $d$-shell. Namely this kind of behavior is known to systematically evade from any DFT-based treatment. The energy expressions of the states in a free $\mathrm{Fe}^{2+}\left(d^{6}\right)$ ion are given in Table I. They nontrivially depend on two Slater-Condon parameters: $F_{2}$ and $F_{4}$. The ground state follows the Hund's rule and for the $\mathrm{Fe}^{2+}\left(\mathrm{d}^{6}\right)$ ion it is the ${ }^{5} D$ state. According to the data published Ref. [36] the states ${ }_{ \pm}^{1} S$ and ${ }_{ \pm}^{1} D$ are not resolved from the spectra. Also the ${ }^{2} F$ state cannot be present in the spectrum of an even-electron system. Thus we exclude three uppermost rows of the Table I and finally arrive to the set of data Table II which can be used for analysis.

To get an impression of what can be (and should be) possibly achieved in terms of describing the 
TABLE II

The excitation energy expressions and their values $\left(\mathrm{cm}^{-1}\right)$ for the many-electron states in the $d$-shell of the $\mathrm{Fe}^{2+}$ ion.

$\begin{array}{llr}\Delta E(-1 G) & 16 . F_{2}+182.5 F_{4}-\frac{1}{2} \sqrt{708 F_{2}^{2}-7,500 F_{2} F_{4}+30,825 F_{4}^{2}} & 30,886.4 \\ \Delta E(+1 G) & 16 . F_{2}+182.5 F_{4}+\frac{1}{2} \sqrt{708 F_{2}^{2}-7,500 F_{2} F_{4}+308,25 F_{4}^{2}} & 57,221.7 \\ \Delta E(1 /) & 6 . F_{2}+180 . F_{4} & 30,356.2 \\ \Delta E(-3 P) & 16 . F_{2}+112.5 F_{4}-\frac{1}{2} \sqrt{912 F_{2}^{2}-9,960 F_{2} F_{4}+38,025 F_{4}^{2}} & 20,688.4 \\ \Delta E(+3 P) & 16 . F_{2}+112.5 F_{4}+\frac{1}{2} \sqrt{912 F_{2}^{2}-9,960 F_{2} F_{4}+38,025 F_{4}^{2}} & 49,576.9 \\ \Delta E(3 D) & 16 . F_{2}+60 . F_{4} & 30,725.8 \\ \Delta E(-3 F) & 16 . F_{2}+112.5 F_{4}-\frac{1}{2} \sqrt{612 F_{2}^{2}-4,860 F_{2} F_{4}+20,025 F_{4}^{2}} & 21,699.9 \\ \Delta E(+3 F) & 16 . F_{2}+112.5 F_{4}+\frac{1}{2} \sqrt{612 F_{2}^{2}-4,860 F_{2} F_{4}+20,025 F_{4}^{2}} & 50,276.1 \\ \Delta E(3 G) & 9 . F_{2}+95 . F_{4} & 24,940.9 \\ \Delta E(3 H) & 4 . F_{2}+120 . F_{4} & 20,300.8\end{array}$

multiple states of the $d$-shells, we determine parameters $F_{2}$ and $F_{4}$ from experimental data. This can be done in a number of ways. The semi-empirical approach is to assume that $F_{2}$ and $F_{4}$ are independent parameters. At the first stage we neglect the correlation and take into consideration only the average energies of the ${ }_{ \pm}^{(2 S+1)} L$ states. The corresponding set of energies is given in Table III. These energies are

\section{TABLE III}

The average multiplet energies $\left(\mathrm{cm}^{-1}\right)$ in the $d$-shell of the $\mathrm{Fe}^{2+}$ ion.

\begin{tabular}{ll}
\hline$E\left({ }_{+}^{1} G\right)_{\mathrm{av}}$ & $44,054$. \\
$\left.E\left({ }^{1}\right)\right)$ & $30,356$. \\
$E\left({ }_{+}^{3} P\right)_{\mathrm{av}}$ & $35,133$. \\
$E\left({ }^{3} D\right)$ & $30,726$. \\
$E\left({ }^{3} F\right)_{a v}$ & $35,988$. \\
$E\left({ }^{3} G\right)$ & $24,941$. \\
$E\left({ }^{3} H\right)$ & $20,301$.
\end{tabular}

linear in the parameters $F_{2}$ and $F_{4}$. Applying the standard linear least squares procedure yields the experimental "noncorrelated" estimate of the parameters (in $\mathrm{cm}^{-1}$ ):

$$
\begin{aligned}
F_{2}^{\exp } & =1,411.0, \\
F_{4}^{\exp } & =120.25, \\
F_{2}^{\exp } / F_{4}^{\exp } & =11.734 .
\end{aligned}
$$

The quality of this result can be assessed by the value of mean square deviation which is 686.80 $\mathrm{cm}^{-1}$ which must be compared with the range of the energies described by the model being about $45,000 \mathrm{~cm}^{-1}$.

Next step consists in estimating the manifestations of correlations in the available data set. The most direct way to do that is to consider the square root contributions to the energies of the multiple terms of the same spin and symmetry. These come 
TABLE IV

The splittings of the multiplets $\left(\mathrm{cm}^{-1}\right)$ with coinciding $L$ and $S$ in the $d$-shell of the $\mathrm{Fe}^{2+}$ ion.

\begin{tabular}{lccc}
\hline & Calc/noncorr & Calc/corr & Exp \\
\hline$\Delta\left({ }_{+}^{1} G\right)$ & 24,140 & 25,984 & $26,335.3$ \\
$\Delta\left({ }_{+}^{3} P\right)$ & 25,993 & 28,255 & $28,888.5$ \\
$\Delta\left({ }_{ \pm}^{3} F\right)$ & 26,142 & 27,726 & $28,576.2$ \\
\hline
\end{tabular}

All values in $\mathrm{cm}^{-1}$.

from the diagonalization of the symmetry adapted CI matrices. Technically, the correlations of that sort are responsible for the splitting within the pairs of states of the same spin and symmetry which do not have any counterpart in the DFT and describe the nontrivial (nondynamical) part of the correlation. We can see from the Table IV that the correlation splitting between the double states is by $\sim 10 \%$ underestimated when calculated with use of the noncorrelated experimental estimates of the $F_{2}^{\exp }$ and $F_{4}^{\exp }$ parameters Eq. (31). The overall picture as coming from the noncorrelated estimate can be characterized by its mean square deviation 1,228.0 $\mathrm{cm}^{-1}$. This fit seems to be improvable by performing another (nonlinear) one for the entire set of available excitation energy expressions and the corresponding experimental values as given in Table II. The result of this new fit is, however, twofold. The resulting values $\left(\mathrm{cm}^{-1}\right)$ of the parameters $F_{2}^{\exp }$ and $F_{4}^{\text {exp }}$ Eq. (32):

$$
\begin{gathered}
F_{2}^{\exp }=1,468.92, \\
F_{4}^{\exp }=113.30, \\
F_{2}^{\exp } / F_{4}^{\exp }=12.960,
\end{gathered}
$$

which can be qualified as "correlated" experimental ones, produce the mean square deviation of 842.37 $\mathrm{cm}^{-1}$ which manifests a significant improvement as compared to analogous usage of the "noncorrelated" experimental values Eq. (31). Meanwhile, although the overall picture is improved, the description of the average multiplet energies is deteriorated when compared with the "noncorrelated" parameters Eq. (31), so that the corresponding mean square deviation somewhat increases to the value of $859.37 \mathrm{~cm}^{-1}$.

The above results deserve thorough attention. First of all, we notice following Refs. [33, 37] that the parameters $F_{2}$ and $F_{4}$ are by definition some functionals of radial density:

$$
\begin{gathered}
F_{k}=\frac{e^{2}}{D_{k}} \int_{0}^{\infty} \int_{0}^{\infty} \frac{\left[\min \left(r_{1}, r_{2}\right)\right]^{k}}{\left[\max \left(r_{1}, r_{2}\right)\right]^{k+1}} R^{2}\left(r_{1}\right) R^{2}\left(r_{2}\right) r_{1}^{2} r_{2}^{2} d r_{1} d r_{2} \\
D_{0}=1 ; \quad D_{2}=49 ; \quad D_{4}=441
\end{gathered}
$$

where $R^{2}(r)$ is the radial density distribution for the involved atomic $d$-shell. However, according to the Theorem 2 of Ref. [14] for whatever spatial multiplet, the one-electron density is spherically symmetric. Thus the quantities $F_{k}$ are the functionals of one-electron density which in the said case have only the radial dependence $r=|\mathbf{r}|$. For that reason, the energies in Table I can be also treated as functionals of the one-electron density representing the averages of the electron-electron interaction energy for each specific many-electron state in the $d$-shell. When supplied by the relevant one-electron contributions (expression for the kinetic energy and that for the electron-nuclear attraction), they become the state specific energy functionals.

$$
\begin{array}{r}
T\left[R^{2}(r)\right]+V_{n e}\left[R^{2}(r)\right]+\frac{n_{d}\left(n_{d}-1\right)}{2} A\left[R^{2}(r)\right] \\
+X C_{n L S}\left[R^{2}(r)\right]
\end{array}
$$

where the contribution proportional to $A\left[R^{2}(r)\right]$ is remarkably analogous to the Hartree energy, however, free from the self-interaction and the $X C_{n L S}$ contributions are the state specific exchange-correlation functionals. They can be treated according to the variational principle (in some analogy with Ref. [37]) this is going to yield some integrodifferential equations for the functions $R(r)$. This option will be considered in details elsewhere. Here, we notice that assuming the model Slater orbital form for the functions $R(r)$ in the $d$-shell:

$$
R(r)=\frac{(2 \zeta)^{n+\frac{1}{2}}}{\sqrt{(2 n) !}} r^{n-1} \exp (-\zeta r)
$$

allows one to evaluate the integrals in Eq. (33) thus leading to the linear dependence of the latter on the orbital exponent $\zeta$ : 


$$
\begin{aligned}
F_{0}^{\text {th }} & =\frac{793}{3072} \zeta \\
F_{2}^{\text {th }} & =\frac{2093 \cdot 5}{49 \cdot 76800} \zeta \\
F_{4}^{\text {th }} & =\frac{91 \cdot 9}{441 \cdot 9216} \zeta
\end{aligned}
$$

(here, $n=3$ ). The ratio of the theoretical values

$$
F_{2}^{\text {th }} / F_{4}^{\text {th }}=\frac{2093 \cdot 5}{49 \cdot 76800} / \frac{91 \cdot 9}{441 \cdot 9216}=13.8
$$

can be compared with one extracted from the correlated and noncorrelated experimental estimates of the parameters $F_{2}^{\exp }$ and $F_{4}^{\exp }$ Eqs. (31) and (32), which indicates that for some reasons the correlated model for the energies better agrees with the Slater model for the radial density distribution. Since as we mentioned the density is spherically symmetric for whatever of the states listed in the above Tables the only parameter characterizing the density is the orbital exponent $\zeta$, provided the said multiplets are constructed on the Slater radial orbitals. In view of the linear dependency of $F_{k}^{\text {th }}$ on $\zeta$ the state specific expressions for the energies and energy differences in Tables I and II become linear functions of $\zeta$ as well.

The excitation energy expressions can be converted to the full scale density functionals for the $d$-shell if one complements the above electron interaction energies by the one-electron terms for the $d$-shell with six electrons in it. The one-electron terms are (i) the kinetic energy per electron:

$$
\frac{\zeta^{2}}{2}
$$

(ii) the potential energy of attraction to the nucleus per electron where the three in the denominator stands for the principal quantum number of the $d$-shell under consideration:

$$
-\frac{Z}{3} \zeta
$$

The average electron-electron interaction value common for all electronic terms is proportional to the Racah $A$ parameter whose expression in terms of $F_{0}^{\text {th }}$ and $F_{4}^{\text {th }}$ is given in the footnote to Table I. For the iron(II) ion, we can set $Z=8$ and the number of $d$-electrons $n_{d}=6$ to take care about the core screening, then the expression for the energy becomes:

$$
3 \zeta^{2}-16 \zeta+15 \frac{143}{576} \zeta .
$$

The value of $\zeta$ comes then as one providing the minimum to the above functional, so that:

$$
\zeta=\frac{8}{3}-\frac{5143}{2576} \approx 2.0460 \cdots
$$

in a remarkable correspondence with the Slater rules yielding for this setting the value of 2.08 simply by ascribing the screening increment of 0.35 to each electron (except one) in the $d$-shell. The screening increment coming form the formula for $A$ amounts 0.387 .

Including further contributions for the electronelectron interaction energy which are now state specific yields for the ground state:

$$
\zeta \approx 2.0621
$$

On the other hand, taking one of the higher excited states ${ }^{1} I$ whose energy is about $30,000 \mathrm{~cm}^{-1}$ above the ground state gives:

$$
\zeta \approx 2.0533
$$

From these estimates, one can derive the following conclusion: The orbital exponent and thus the radial density is very weakly sensitive to whatever correlations. This finding is in agreement both with the accepted concept of correlation which attributes it exclusively to the cumulant of the two-electron density matrix so that there is no need to reload its manifestations on the density as well as with numerous demonstrations of no relation between the one-electron density and the correlations known in the literature (see e.g., Ref. [38]).

Further analysis can be based on the observation that inserting the theoretical definitions for the $F_{2}^{\text {th }}$ and $F_{4}^{\text {th }}$ parameters Eq. (33) into expressions for the excitation energies result in linear models for these energies with the single fitting parameter $\zeta$. Two such models can be constructed: the noncorrelated which uses only the average energies of multiple states with equal $L$ and $S$ and the correlated one which covers all 10 available excitation energies. Fitting the excitation energies to the noncorrelated model yields the value of 2.4823 for $\zeta$. The quality of 
fitting with only one parameter is certainly somewhat worse than that using two independent parameters $F_{2}^{\exp }$ and $F_{4}^{\exp }$ and the mean square deviation becomes $1,058.0 \mathrm{~cm}^{-1}$ for the set of average energies of the multiplets (noncorrelated fit). The value of $\zeta$ which comes from the linear fitting procedure with the correlated energy expressions is 2.4604 and the mean square deviation is $1,013.3$ $\mathrm{cm}^{-1}$. We see that also in this case the correlations only marginally affect the one-electron density distribution and that despite some deterioration of the precision as compared with the two-parameter models the overall quality of the fit is surprisingly good.

\subsection{SUMMARY}

Let us summarize the findings of this Section. We managed to obtain simple expressions for the energies of the nontrivially correlated ionic states (these expressions include nondynamic correlation through the square root terms) with definite values of $L$ and $S$ as functions of a single parameter $\zeta$, the Slater orbital exponent for the $d$-shell. In the context of the accepted model, it is the only quantity characterizing the density in the $d$-shell. In a sense, there is one-to-one correspondence between the electron density of the $d$-shell and $\zeta$ thus the expressions for the energy can be considered as state specific energy functionals of the form:

$n_{d} \frac{\zeta^{2}}{2}-n_{d} \frac{Z}{n} \zeta+\frac{n_{d}\left(n_{d}-1\right)}{2} \frac{143}{576} \zeta+X C_{n L S}\left(n_{d}, \zeta\right)$

written in terms of the orbital exponent $\zeta$ uniquely related to the density within the model used and where $\mathrm{XC}_{n L S}\left(n_{d}, \zeta\right)$ stands for state dependent exchange-correlation terms as obtained by inserting the expressions for the Slater-Condon parameters $F_{k}^{\text {th }}$ Eq. (35) in the expressions given in Table I or analogous expressions for other $d$-shell fillings Ref. [34].

As one can see our estimates of the characteristic quantity $\zeta$ yield the values which fall into two classes depending on the type of the estimate, those coming from the variational estimate for the total energy of each respective state give the values close to $\zeta=2.08$ coming from the Slater rules. The estimates based on fitting the excitation energies to $\zeta$ yield much larger value (much less diffuse $d$-shell) about 2.5 with extremely weak influence of electron correlation on the estimates of either of these types.
These numerical results must be compared with other (empirical) values of the orbital exponents. These, however, demonstrate a wide range of values. For example, Ref. [39] report the value $\zeta=$ 3.7266; Ref. [40] suggests $\zeta=3.152$; Ref. [41] gives $\zeta$ $=2.722$; and Ref. [42] provides $\zeta=3.15$ basically repeating the value of Ref. [40]. This indicates that either the correlated or noncorrelated estimates, coming from the excitation energies only, fall in the range defined by the Slater rules and other semiempirical estimates. Although, the deviations between the density parameter estimates coming from different types of procedures also are expectable (we remind the existence of distinct thermochemical and spectral semi-empirical parameterizations) the true source of observed deviations is of certain interest.

\section{Range-Separated Treatment of Electronic Coulomb Interaction in Atomic d-Shells}

Based on the idea that the short-range behavior of the e-e interactions can be efficiently transferred from the homogeneous e-gas to arbitrary manyelectron systems, whereas the long-range e-e interactions being much more system specific (less transferable), Savin and Stoll suggested a generalization of the Kohn-Sham theory by splitting explicitly the short- and long-range e-e interactions [4346]. The nontransferable long-range interactions can be assimilated to a wave function treatment, just like the kinetic energy in the conventional Kohn-Sham model, which results in a replacement of the noninteracting Kohn-Sham reference system by a "long-range-interacting" one. Although in conventional KS theory, the effective KS Hamiltonian has an exact single-determinant solution, the generalized, range-separated variant includes a certain amount of explicit e-e interaction and the corresponding effective Schrödinger equation has to be solved in a multideterminant form [47]. However, due to the nonsingular nature of the $1 r$ Coulomb operator, the solution can be converged considerably faster in both the one-electron and many-electron basis.

Recent works on the range-separated hybrid methods were mostly based on the separation of the Coulomb potential into short- and long-range parts was performed according to: 


$$
\begin{aligned}
\frac{1}{r_{12}} & =\left(\frac{1}{r_{12}}\right)_{s}+\left(\frac{1}{r_{12}}\right)_{l} \\
\left(\frac{1}{r_{12}}\right)_{s} & =\frac{\operatorname{erf}\left(\mu r_{12}\right)}{r_{12}} \\
\left(\frac{1}{r_{12}}\right)_{l} & =\frac{\operatorname{erfc}\left(\mu r_{12}\right)}{r_{12}} \\
1 & =\operatorname{erf}(x)+\operatorname{erfc}(x)
\end{aligned}
$$

The treatment of the long-range exchange has been done in the Hartree-Fock framework, while the correlation could be treated by MP2 [48] or $\operatorname{CCSD}(\mathrm{T})[49]$ level, leading to a successful description of London dispersion forces in $\mathrm{vdW}$ complexes [50] or by MCSCF level [51] to treat typical nondynamic correlation problems, like the case of the $\mathrm{H}_{2}$ dissociation. A simpler model, where long-and short-range correlations are both handled by density functional approximations (RSHX-exchangeonly range separated hybrid [52], like LC- $\omega$ PBE of Scuseria [53]) has been recently shown to be quite successful in predicting magnetic coupling constants in transition metal systems [54].

In the following, we examine the behavior of the range-separated approach on the simple Fe(II) ion model system.

\subsection{RANGE SEPARATED HYBRID APPROACH}

To make easier the evaluation of analytical integrals and obtain the $F_{k}^{\text {th }}$ parameters, we decided to employ the "Yukawa"-like separation as proposed in Ref. [55]:

$$
\begin{aligned}
& \left(\frac{1}{r_{12}}\right)_{s}=\frac{\exp \left(-\beta r_{12}\right)}{r_{12}} \\
& \left(\frac{1}{r_{12}}\right)_{l}=\frac{1-\exp \left(-\beta r_{12}\right)}{r_{12}} .
\end{aligned}
$$

The value of $\beta \rightarrow 0$ corresponds to the absence of the long-range part. By contrast $\beta \rightarrow \infty$ corresponds to the evanescence of the short-range part. The reach of the short-range interactions is roughly inversely proportional to the value of $\beta$ measured in inverse bohr units.

The initial assumption is that only the long-range part of the Coulomb interaction contributes to the non-dynamical correlations in the $d$-shells so that only the matrix elements of $\left(1 / r_{12}\right)_{l}$ must be taken into account when the CI matrices describe the nontrivial correlation in the $d$-shells. To check this assumption, we have performed the following. With use of analytical results of Refs. [56, 57] we get for the Yukawa potential the following expansion:

$$
\begin{gathered}
\left|\mathbf{r}_{1}-\mathbf{r}_{2}\right|^{-1} \exp \left(-\beta\left|\mathbf{r}_{1}-\mathbf{r}_{2}\right|\right)=4 \pi \sum_{l=0 m=-1}^{\infty} \sum^{l}\left(r_{<} r_{>}\right)^{-\frac{1}{2}} \\
I_{l+\frac{1}{2}}\left(\beta r_{<}\right) K_{l+\frac{1}{2}}\left(\beta r_{>}\right) Y_{l}^{-m}\left(\frac{r_{<}}{r_{<}}\right) Y_{l}^{m}\left(\frac{r_{>}}{r_{>}}\right)
\end{gathered}
$$

where $I_{l+\frac{1}{2}}$ and $K_{l+\frac{1}{2}}$ are the modified Bessel functions of the half-integer index, $r_{<}=\min \left(r_{1}, r_{2}\right), r_{>}$ $=\max \left(r_{1}, r_{2}\right)$, and the vectors $\mathbf{r}_{<}$and $\mathbf{r}_{>}$are assigned correspondingly. The above expression must be inserted in the definition of the matrix elements of the electron-electron interaction (see e.g., Ref. [34]) which due to the spherical symmetry of the Yukawa potential allows us to express these latter in terms of the short range analogs of the Slater-Condon parameters. For the $3 d$ Slater orbitals with the orbital exponent $\zeta$, the estimates for the short range $F_{2}^{(\mathrm{s})}$ and $F_{4}^{(\mathrm{s})}$ and long-range $F_{2}^{(1)}$ and $F_{4}^{(1)}$ contributions to the $F_{k}^{\text {th }}$ parameters Eq. (35) are:

$$
\begin{gathered}
F_{2}^{(s)}=F_{2} f^{(s)} ; \quad F_{2}^{(1)}=F_{2} f^{(1)} ; \quad f^{(1)}+f^{(s)}=1 \\
F_{4}^{(s)}=F_{4} g^{(s)} ; \quad F_{4}^{(1)}=F_{4} g^{(1)} ; g^{(1)}+g^{(s)}=1 \\
f^{(1)}=1-\frac{4 \zeta^{2}}{6,279(\beta+2 \zeta)^{12}}\left(1,575 \beta^{10}+37,800 \beta^{9} \zeta\right. \\
+413,420 \beta^{8} \zeta^{2}+2,714,880 \beta^{7} \zeta^{3}+11,850,720 \beta^{6} \zeta^{4} \\
+35,848,960 \beta^{5} \zeta^{5}+75,603,840 \beta^{4} \zeta^{6} \\
+107,827,200 \beta^{3} \zeta^{7}+94,591,744 \beta^{2} \zeta^{8} \\
\left.+38,578,176 \beta \zeta^{9}+6,429,696 \zeta^{10}\right) \\
g^{(1)}=1-\frac{4 \zeta^{2}}{91(\beta+2 \zeta)^{12}\left(63 \beta^{10}+1,512 \beta^{9} \zeta\right.} \\
+16,380 \beta^{8} \zeta^{2}+104,832 \beta^{7} \zeta^{3}+433,888 \beta^{6} \zeta^{4} \\
+1,188,096 \beta^{5} \zeta^{5}+2,101,632 \beta^{4} \zeta^{6} \\
+2,263,040 \beta^{3} \zeta^{7}+1,487,616 \beta^{2} \zeta^{8}+559,104 \beta \zeta^{9} \\
\left.+93,184 \zeta^{10}\right) \quad(47)
\end{gathered}
$$

Introducing a new variable 


$$
t=\frac{\beta}{\beta+2 \zeta}
$$

we get somewhat simpler expressions for the longrange scaling coefficients,

$$
\begin{array}{r}
g^{(1)}=-\frac{256 t^{12}}{91}+\frac{2304 t^{11}}{91}-\frac{8,832 t^{10}}{91}+\frac{18,432 t^{9}}{91} \\
-240 t^{8}+144 t^{7}-16 t^{6}-\frac{144 t^{5}}{7}-\frac{6 t^{4}}{7}+\frac{30 t^{3}}{7} \\
+\frac{15 t^{2}}{7} \\
f^{(1)=}-\frac{6,400 t^{12}}{6,279}+\frac{75,520 t^{11}}{6,279}-\frac{1,34,528 t^{10}}{2,093} \\
+\frac{1,285,120 t^{9}}{6,279}-\frac{384,880 t^{8}}{897}+\frac{14,160 t^{7}}{23}-\frac{23,600 t^{6}}{39} \\
+\frac{2,451,808 t^{5}}{6,279}-\frac{23,150 t^{4}}{161}+\frac{6910 t^{3}}{483}+\frac{3,455 t^{2}}{483}
\end{array}
$$

Numerical optimization of the sum of square deviations, where the theoretical values are obtained under the condition that the parameters $F_{2}$ and $F_{4}$ under the square roots are, respectively, replaced by the long range contributions $F_{2}^{(1)}$ and $F_{4}^{(1)}$, with respect to $\zeta$ and $t$ results in the values:

$$
\begin{aligned}
\zeta & =2.46425 \\
t & =0.725436 \\
\beta & =13.0218
\end{aligned}
$$

The range separation parameter $\beta$ is obtained by inverting the definition of $t$. These values correspond to the following scaling parameters:

$$
\begin{aligned}
& f^{(1)}=0.965879 \\
& g^{(1)}=0.912733
\end{aligned}
$$

The precision of this estimate can be characterized as previously by the mean square deviation which amounts to $996 \mathrm{~cm}^{-1}$. Taking into account that the short-range e-e potential corresponding to $\beta=13.02$ falls down to a negligibly small value, say 0.001 , for $r=0.5 \mathrm{bohr}$, it can be concluded that electron repulsion at shorter than 0.5 bohr direct space distance has an insignificant effect on the multiplet structure.

By contrast, if the theoretical values are obtained under the condition that the parameters $F_{2}$ and $F_{4}$ under the square roots are, respectively, replaced by the short range contributions $F_{2}^{(\mathrm{s})}$ and $F_{4}^{(\mathrm{s})}$, the optimization of the sum of square deviations with respect to $\zeta$ and $t$ results in the values:

$$
\begin{aligned}
\zeta & =2.4653 \\
t & =0.0482271 \\
\beta & =0.249838
\end{aligned}
$$

These values correspond to the magnitudes of the scaling parameters:

$$
\begin{aligned}
& f^{(s)}=0.982441 \\
& g^{(s)}=0.994545
\end{aligned}
$$

Incidentally, the precision of the procedure singling the short-range part characterized as previously by the mean square deviation yields the value $985 \mathrm{~cm}^{-1}$, quite similar to the long-range estimate. The reach of the "short- range" interactions, measured by analogous criteria as before (falling off the short-range Coulomb potential below 0.001) is about $r=16 \mathrm{bohr}$, which englobes practically the full range for the significant densities of the $d$ electrons. It means that the long-range "tail" of the electron-electron interactions is essential to recover the correct muliplet structure. Furthermore, one can see that the renormalization of the $F$ functions is in the order of $1 \%$, confirming that the use of the optimal $\beta$ implies the involvement of practically the full range of interactions (cf., previous Section).

A further lesson drawn from this simple model study is that the short range/long range separation of the Coulomb potential is not sensitive to the correlations as well: the characteristic parameter of the density distribution $\zeta$ in all cases equals to 2.46 with variations in the third digit after the decimal point. Thus the short range/long range separation does not lift thus the strong contradiction between the estimates of the orbital exponent by the Slater rules or variationally from the state specific functionals Eq. (43) and those from linear fit for the excitation energies. Thunkable way out looks out twofold: First, the Slater rules can be thought to overestimate the screening (for the $d$-shell the screening by the inner shells is treated to be com- 
plete, which yields the value 8 for the effective charge) thus leading to the values of $\zeta$ too small when compared with those extracted from the fitting of the experimental data on excitation energies. Second, one can think that the value of parameter $F_{0}$ is for some reason much stronger renormalized when compared with its theoretical value Eq. (35) than those of the parameters $F_{2}$ and $F_{4}$. If we apply long-short range separation and calculate $F_{0}$ at the values of $\beta$ and $\zeta$ Eq. (52) extracted from the fitting of excitation energies with the short-range parts $F_{2}^{(\mathrm{s})}$ and $F_{4}^{(s)}$ under the square roots the fraction of the short range part in $F_{0}$ amounts $h^{(\mathrm{s})}=0.688608$ of the latter. Now, if we assume that the short range part for some reason renormalizes to zero and thus only the long range part of the e-e potetial contributes to the real value of $F_{0}$ then the variational estimate of the orbital exponent reads:

$$
\zeta=\frac{8}{3}-\frac{5}{2} \cdot \frac{143}{576} \cdot\left(1-h^{(\mathrm{s})}\right) \approx 2.4743
$$

which shows some reasonable consistency with the values extracted from analysis of experimental spectra. Of course, this may well be a pure coincidence, but possible consequences of the above hypothesis on the way of renormalization of the Slater-Condon parameters will be considered elsewhere.

\section{Conclusion}

In this article, we discussed a few possible ways of avoiding the dead-locks of the pragmatic methods of molecular electronic structure theory based on the DFT, which appear due to the non-sensitivity of the basic quantity of the DFT, the one-electron density, to the differences in the spin (permutational) or/and spatial symmetry of the underlying many-electronic states. This nonsensitivity is reflected by two theorems (recent Theorems 1 and 2 of Ref. [14]) which formalize two basically known facts that (i) the one-electron density does not depend on the total spin of the many-electron state, and, that (ii) the one-electron density in a manyelectronic state, which transforms according to any irreducible representation of the group acting on the spatial coordinates of electrons $(S O(3), S O(2)$, or their point subgroup), transforms according the fully symmetric irreducible representation of the corresponding group.
These theorems imply necessarily that the information concerning the symmetry of the respective many-electronic states at hand is to be introduced into any DFT-based treatment extraneously. When it goes about the total spin (or equivalently about the permutational symmetry) of a many-electron state, we suggest to use statespecific functionals labeled by the Young tableaux $Y \nu$ (the rows of the irreducible representations of the unitary group $U(N)$ ) and to develop a procedure analogous to ROKS for each of them. In the particular case of multiple states sharing the same $L$ and $S$ in the $d$-shells of transition metal ions, we suggest state-specific correlated functionals of the density and their model based on the assumption of a Slater orbital form of the radial density distribution. This procedure reduces the functional-type density dependence to function-type dependence on the orbital exponent. With the use of these expressions the excitation energies of the many-electron states of the $\mathrm{Fe}^{2+}$ ion are reproduced with remarkable accuracy. The variational treatment of the proposed functionals reproduces with similar precision the values of the orbital exponent of the $\mathrm{Fe}^{2+}$ ion prescribed by the Slater rules. Nevertheless, the estimates of the orbital exponent coming from the variational principle and from the fit of the excitation energies differ significantly although they fall in the range provided by different semi-empirical estimates. Some ideas related to conciliation of these two groups of estimates have been derived from analysis of the short range/long range separation of the electron-electron interaction potential.

\section{ACKNOWLEDGMENTS}

The authors are thankful to Profs. I. V. Abarenkov, I. Mayer, G. M. Zhidomirov, I. G. Kaplan, A. Savin, J.-P. Malrieu, Á. Nagy for valuable discussions. ALT expresses thanks to Miss O. A. Tchougréeva for her assistance.

\section{Appendix: Permutation Symmetry of the Spatial Function}

Because the Hamiltonian does not depend on spin variables one may wonder why the total spin at all affects the energy. The answer lays in the symmetry of many-electron wave functions with 
respect to permutations of coordinates $x_{i}$ of all $N$ electrons of the system (group $S_{N}$ ). The correct wave function must be antisymmetric with respect to them (Pauli principle for fermions). This simple statement applies when the complete electronic coordinates $x_{i}=\left(\mathbf{r}_{i}, s_{i}\right) ; i=1 \div N$ are taken as arguments of the wave function. Because of the fact that the nonrelativistic electronic Hamiltonian does not depend on the spin projections $s_{i}$ the wave function of electrons can be represented as a product of the spatial and spin parts dependent respectively on the spatial $\left(\mathbf{r}_{i}\right)$ and spin $\left(s_{i}\right)$ coordinates only (see e.g., Eq. (10)) with the antisymmetry requirement applicable to the entire products. To calculate the energy, it is enough to know only the spatial part (multiplier) of the $\mathrm{N}$-electronic wave function. As one can see in Eq. (10) the spatial parts of the triplet and the singlet are, respectively, antisymmetric and symmetric with respect to permutations of the spatial coordinates $\mathbf{r}_{1}$ and $\mathbf{r}_{2}$ and namely this difference is the only real source of the differences in the energy.

In contrast with the simple permutation symmetry properties of the complete wave functions those of the spatial multipliers are in general case somewhat more involved. The permutation properties are conveniently described in terms of the Young patterns and Young tableaux. Generally, the Young patterns are shapes formed by $N$ boxes arranged in rows of non-increasing length:

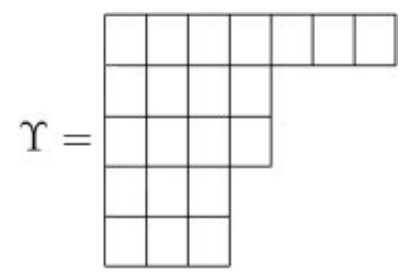

These shapes label the irreducible representations of the group $S_{N}$. The Young pattern corresponding to the totally antisymmetric wave function contains only one column of the height $N$. The fact that there is only one possibility to fill this column by electron labels from 1 to $N$ corresponds to the one-dimensionality of the antisymmetric representation of the $S_{N}$ group. Separation of the antisymmetric function into spatial and spin parts predefines their respective permutation properties: they must belong to the adjoint representations of the $S_{N}$ group, because the product of two functions belonging to adjoint representations yields the required antisymmetric function. The Young patterns corresponding to adjoint representa- tions of $S_{N}$ are connected by $180^{\circ}$ rotation around the bissectriss of their common upper left corner. Because the Young patterns which can be used for constructing the spin functions may contain no more than two rows, those usable for constructing the electronic spatial functions, respectively, cannot contain more than two columns:
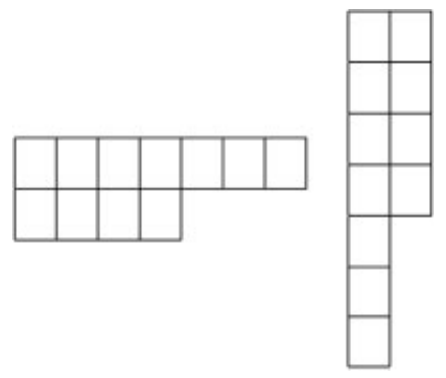

The most remarkable feature of the Young patterns as applied to electronic wave functions is that they are in a one-to-one correspondence with the total spin, namely: the length of the one-column part of the spatial Young pattern equals to $2 S$. This allowed F. A. Matsen [23] yet many years ago to suggest to avoid any remark concerning the spin in (nonrelativistic) quantum chemistry context and to replace it by referencing to the permutational symmetry of the corresponding states. Although, it is, of course, a matter of terminology, within such a formulation the triplet component with $S_{z}=+1$ would never arise by this, lifting any possible confusion.

The same tools can be used to describe the irreducible representations of the group of unitary matrices. The corresponding construct evolves as follows Ref. [18]: for any number of spatial orbitals $M$ the group $U(M)$ of the unitary $M \times M$ matrices acts as a "dynamical" group by transforming orbitals. Any given number of electrons $N$ and any value of the total spin $S$ conforming with two previous values produces an irreducible representation $Y$ of the group $U(M)$. As in the case of the $S_{N}$ group irreducible representations of the group $U(M)$ are labeled by the Young patterns, but the meaning of their elements is different. The representation by $\mathrm{N}$-electron spatial functions has the tensor rank $N$ and the corresponding Young pattern contains $N$ boxes arranged in no more than two columns each of the height not larger than $M$, such that the first column is by $2 S$ boxes longer than the second one. This irreducible representation is degenerate and its rows $v$ can be numbered by distributing $M$ orbital symbols in the above $N$ boxes in such a way that 
they do not decrease (some ordering is assumed among them) along the rows and strictly increase in each column. Under this rule, some orbital symbols in principle may appear no more than twice in a two-column pattern by this representing a doubly occupied spatial orbital, those appearing once represent singly occupied orbitals. Thus constructed Young tableaux represent states transforming according to the rows $\nu$ of the representation $Y$. The Young tableau characterizes first of all the permutation symmetry of the state in that sense that the spatial part of the many electron function described by the Young tableau $Y \nu$ is derived from the product of orbitals where each enters as many times as it appears in the tableau by applying the symmetrization over rows of the tableau and antisymmetrization over its columns. This construct is also known as immanant wave functions Ref. [27].

\section{References}

1. Parr, R. G.; Yang, W. T. Density-Functional Theory of ATOMS and Molecules; Oxford University Press: New York, 1994.

2. March, N. H. Electron Density Theory of Atoms and Molecules; Academic Press: New York, 1992.

3. Koch, W.; Holthausen, M. C. A Chemist's Guide to Density Functional Theory; Wiley-VCH: Weinheim, New York, 2001.

4. Darkhovskii, M.; Tchougréeff, A. In Progress in Theoretical Chemistry and Physics; Julien, J.-P.; Maruani, J.; Brändas, E., Eds.; Springer: New York, 2006; p 451.

5. Hohenberg, P.; Kohn, W. Phys Rev 1964, 136, 864.

6. Blokhintsev, D. Quantum Mechanics; Springer: New York, 1994.

7. Kohn, W.; Sham, L. J. Phys Rev 1965, 140, A1133.

8. Löwdin, P. O. Adv Chem Phys 1959, 2, 207.

9. Kutzelnigg, W. Theor Chim Acta 1993, 86, 41.

10. Kutzelnigg, W. Int J Quantum Chem 2003, 95, 404.

11. Fritsche, L.; Koller, J. J Solid State Chem 2003, 176, 652.

12. Ziesche, P. Int J Quantum Chem 2002, 90, 342.

13. Zülicke, L. Quantenchemie; Deutscher Verlag der Wissenschaften: Berlin, 1973; Vol. 1.

14. Kaplan, I. G. J Mol Struct (Theochem) 2007, 838, 39.

15. Kaplan, I. G. Int J Quantum Chem 2007, 107, 2595.

16. Abarenkov, I. V.; Zagoulaev, S. N. Int J Quantum Chem 2008, 108, 2657.

17. Ziegler, T.; Rauk, A.; Baerends, E. J. Theor Chem Acc 1977, 43, 261.

18. McWeeny, R. Methods of Molecular Quantum Mechanics; Academic Press: London, 1992.

19. Slater, J. C. Adv Quantum Chem 1972, 6, 1.

20. Russo, T. V.; Martin, R. L.; Hay, P. J. J Chem Phys 1994, 101, 7729.

21. Filatov, M.; Shaik, M. Chem Phys Lett 1998, 288, 689.
22. Roothaan, C. C. J. Rev Mod Phys 1960, 32, 179.

23. Matsen, F. A. Adv Quantum Chem 1964, 1, 59.

24. (a) Goddard, W. A., III. Phys Rev 1967, 157, 73; (b) Goddard, W. A., III. J Chem Phys 1968, 48, 450; (c) Goddard, W. A., III. J Chem Phys 1968, 48, 5337.

25. Vojtik, J.; Kvasnièka, V. Phys Rev A 1972, 5, 1568.

26. Sullivan, J. Phys Rev A 1972, 5, 29.

27. Poshusta, R.; Kramling, R. Phys Rev 1968, 167, 139.

28. von Barth, U. Phys Rev A 1979, 20, 1693.

29. Gunnarsson, O.; Lundqvist, B. I. Phys Rev B 1976, 13, 4274.

30. Rosa, A.; Ricciardi, G.; Gritsenko, O.; Baerends, E. J. In Structure and Bonding; Kaltsoyannis, N.; McGrady, J., Eds.; Springer: New York, 2004; p 49.

31. Kaplan, I. G. Symmetry of Many-Electron Systems; Academic Press: New York, 1975.

32. (a) Nagy, Á. Int J Quantum Chem 1998, 70, 681; (b) Nagy, Á. J Phys B: At Mol Opt Phys 1999, 32, 2841.

33. Nagy, Á.; Liu, S.; Bartolloti, L. J Chem Phys 2005,122,134107.

34. Ballhausen, C. Introduction to Ligand Field Theory. McGraw-Hill Book: New York, 1962; p 6.

35. Sviridov, D.; Smirnov, Y. Theory of Optical Spectra of Transition-Metal Ions; Nauka: Moscow, 1977.

36. http://www.nist.gov.

37. Hamamoto, N.; Satoko, C. J Phys B 2004, 37, 4309.

38. Eschrig, H. The Fundamentals of Density Functional Theory; Teubner, 1996.

39. Clementi, E.; Raimondi, D. J Chem Phys 1963, 38, 2686.

40. Burns, D. J Chem Phys 1964, 41, 1521.

41. Gouterman, M.; Zerner, M. Theor Chem Acc 1966, 4, 44.

42. Böhm, M.; Gleiter, R. Theor Chem Acc 1981, 59, 127.

43. Stoll, H.; Savin, A. Density Functional Methods in Physics; Dreizler, R. M.; Providencia, J. D., Eds.; Plenum: New York, 1985; p 177.

44. Savin, A. Recent Developments ans Applications of Modern Density Functional Theory; Seminario, J. M., Ed.; Elsevier: Amsterdam, 1996; p 327.

45. Savin, A. Recent Advances in Density Functional Theory; Chong, D. P., Ed.; World Scientific: Singapore, 1996; p 129.

46. Leininger, T.; Stoll, H.; Werner, H.-J.; Savin, A. Chem Phys Lett 1997, 275, 151.

47. Toulouse, J.; Colonna, F.; Savin, A. Phys Rev A 2004, 70, 062505.

48. Ángyán, J. G.; Gerber, I. C.; Savin, A.; Toulouse, J. Phys Rev A 2005, 72, 012510.

49. Goll, E.; Werner, H.-J.; Stoll, H. Phys Chem Chem Phys 2005, 7, 3917.

50. Gerber, I. C.; Ángyán, J. G. J Chem Phys 2007, 126, 044103.

51. Fromager, E.; Toulouse, J.; Jensen, H. J. A. J Chem Phys 2007, 126, 074111.

52. Gerber, I. C.; Ángyán, J. G. Chem Phys Lett 2005, 415, 100.

53. Vydrov, O. A.; Scuseria, G. E. J Chem Phys 2006, 125, 234109.

54. Rivero, P.; de, P. R.; Moreira, I.; Illas, F.; Scuseria, G. E. J Chem Phys 2008, 129, 184110.

55. Savin, A.; Flad, H.-J. Int J Quantum Chem 1995, 56, 327.

56. Steinborn, E.; Filter, E. Theor Chim Acta 1975, 38, 261.

57. Steinborn, E.; Filter, E. Theor Chim Acta 1975, 38, 273. 